\title{
Therapeutic drug monitoring of oral targeted antineoplastic drugs
}

\author{
Anna Mueller-Schoell ${ }^{1,2} \cdot$ Stefanie L. Groenland ${ }^{3} \cdot$ Oliver Scherf-Clavel $^{4} \cdot$ Madelé van Dyk $^{5} \cdot$ Wilhelm Huisinga $^{6}$. \\ Robin Michelet ${ }^{1} \cdot$ Ulrich Jaehde $^{7} \cdot$ Neeltje Steeghs $^{3} \cdot$ Alwin D.R. Huitema ${ }^{8,9} \cdot$ Charlotte Kloft $^{1}$ (1)
}

Received: 2 July 2020 / Accepted: 1 October 2020 / Published online: 9 November 2020

(C) The Author(s) 2020, corrected publication 2020

\begin{abstract}
Purpose This review provides an overview of the current challenges in oral targeted antineoplastic drug (OAD) dosing and outlines the unexploited value of therapeutic drug monitoring (TDM). Factors influencing the pharmacokinetic exposure in OAD therapy are depicted together with an overview of different TDM approaches. Finally, current evidence for TDM for all approved OADs is reviewed.

Methods A comprehensive literature search (covering literature published until April 2020), including primary and secondary scientific literature on pharmacokinetics and dose individualisation strategies for OADs, together with US FDA Clinical Pharmacology and Biopharmaceutics Reviews and the Committee for Medicinal Products for Human Use European Public Assessment Reports was conducted.

Results OADs are highly potent drugs, which have substantially changed treatment options for cancer patients. Nevertheless, high pharmacokinetic variability and low treatment adherence are risk factors for treatment failure. TDM is a powerful tool to individualise drug dosing, ensure drug concentrations within the therapeutic window and increase treatment success rates. After reviewing the literature for 71 approved OADs, we show that exposure-response and/or exposure-toxicity relationships have been established for the majority. Moreover, TDM has been proven to be feasible for individualised dosing of abiraterone, everolimus, imatinib, pazopanib, sunitinib and tamoxifen in prospective studies. There is a lack of experience in how to best implement TDM as part of clinical routine in OAD cancer therapy.

Conclusion Sub-therapeutic concentrations and severe adverse events are current challenges in OAD treatment, which can both be addressed by the application of TDM-guided dosing, ensuring concentrations within the therapeutic window.
\end{abstract}

Keywords Targeted antineoplastic drugs · Tyrosine kinase inhibitors - Therapeutic drug monitoring · Oral anticancer drugs · Personalised medicine

The original article was revised: Due to an error during production, some intext and most of the in-table citations were not correctly displayed in the original previous online version of the article and have now been corrected.

Supplementary Information The online version of this article (https:// doi.org/10.1007/s00228-020-03014-8) contains supplementary material, which is available to authorized users.

Charlotte Kloft

charlotte.kloft@fu-berlin.de

1 Dept. of Clinical Pharmacy and Biochemistry, Institute of Pharmacy, Freie Universitaet Berlin, Berlin, Germany

2 Graduate Research Training Program, PharMetrX, Berlin/ Potsdam, Germany

3 Department of Clinical Pharmacology, Division of Medical Oncology, The Netherlands Cancer Institute-Antoni van Leeuwenhoek, Plesmanlaan 121, 1066

CX Amsterdam, The Netherlands
4 Institute of Pharmacy and Food Chemistry, Julius-Maximilians-Universität Würzburg, Würzburg, Germany

5 College of Medicine and Public Health, Flinders University, Adelaide, SA, Australia

6 Institute of Mathematics, University of Potsdam, Potsdam, Germany

7 Department of Clinical Pharmacy, Institute of Pharmacy, University of Bonn, Bonn, Germany

8 Department of Pharmacy \& Pharmacology, The Netherlands Cancer Institute-Antoni van Leeuwenhoek, Amsterdam, The Netherlands

9 Department of Clinical Pharmacy, University Medical Center, Utrecht University, Utrecht, The Netherlands 


\section{Introduction}

With the approval of imatinib in 2001 [1], kinase inhibitors (KIs) have significantly improved the prognosis of many cancers. As of April 2020, 71 oral antineoplastic drugs (OADs) targeting a large assortment of molecular targets (Supplementary Fig. 1) are approved by the European Medicines Agency (EMA) and/or the US Food and Drug Administration (FDA).

With more OADs available, both the route of administration and the treatment setting are changing. While i.v. chemotherapy is mainly administered in an in-patient setting, OADs allow outpatient care with both its advantages and disadvantages. Strong advantages are the level of independence, and, due to outpatient treatment, a reduction of health care costs. At the same time, the responsibility for adhering to treatment schedules is moved to the patient. Given the often-complex treatment regimens, patients must be well trained and motivated to take their medication correctly. Moreover, patients should have knowledge on the frequency and severity of possible adverse events (AEs) and on preventive and responsive measures to limit them. Yet, adherence to targeted OADs is variable.

Other aspects to consider are the complex pharmacokinetics (PK) of OADs [2]. Although the right drug ('what') is increasingly selected based on the tumour characteristics, a fixed dose ('how much') is mostly given in OADs, leading to large differences between individual plasma concentrations. High interindividual variability (IIV) in exposure at standard dosing, mostly ranging from 19 to $100 \%$ [3] and up to 16-fold for gefitinib [4], has been described for OADs. While modern phase I studies increasingly assess exposure-response relationships and maximum tolerated doses (MTD) become harder to identify, the fixed dose for a new antineoplastic drug is historically established in a phase I study using a $3+3$ design, which focuses on toxicity [5]. The MTD, defined as the dose level below the toxic dose level, is usually adopted as the recommended phase II dose [5]. Few patients participate in phase I trials (median $n=26$ [6]) which limits the generalisability of the selected dose. Based on the lack of focus on efficacy, a proportion of patients will show subtherapeutic plasma concentrations [7] and be at risk for treatment failure at the early determined MTD. At the same time, some patients will show toxic plasma concentrations and thus an increased risk for non-adherence [8] as consequence of AEs [9].

One strategy to prevent sub-optimal drug concentrations is the use of therapeutic drug monitoring (TDM), i.e. dosing based on measured drug exposure [10], guiding OAD dosing [7, 11-14]. By tailoring drug doses to individual patients, the proportion of patients with sub-optimal drug concentrations can be reduced. TDM has already been well-adopted in other therapeutic areas such as antimicrobial and antiepileptic therapy [15-17]. Despite its value in oncology becoming more recognised [18-21], it is still not commonly used in antineoplastic treatment.

In the following sections, we elaborate on the unexploited value of TDM in OAD therapy. After introducing various forms of TDM and TDM for OADs specifically, an overview of current evidence for drug target concentrations is provided. Moreover, we describe available PK models, observed PK exposure, TDM targets and data on exposure-response and exposure-safety relationships for OADs that are approved by at least one regulatory agency. Finally, TDM recommendations are given for OADs, for which targets were established and TDM has proven feasible.

\section{Therapeutic drug monitoring}

Therapeutic drug monitoring (TDM) refers to measuring drug concentrations to assess if drug concentrations are within the therapeutic target range and, if necessary, individualise dosing regimens. An unpredictable dose-exposure relationship, a small therapeutic window with a defined target concentration, a high PK and/or pharmacodynamic (PD) IIV and nonlinear PK are best indicators for a benefit from TDM [7, 22]. The absence of an exposure-response relationship and high intraindividual and interoccasion (IOV) $\mathrm{PK} / \mathrm{PD}$ variability relative to the IIV are characteristics of drugs unsuitable for TDM [23, 24] (Supplementary Fig. 2). Several forms of dose individualisation exist. These are classified as a priori and a posteriori approaches, depending on the level of individualisation before treatment initiation [15]. In an a priori framework, information on both drug and patient characteristics are used to guide initial dosing [25]. Based on established relationships between patient characteristics and PK parameters, initial dosing can be individualised to patient subpopulations [26]. However, no individual PK information is included in an a priori framework, resulting in moderate average bias and precision [26].

Individual drug concentrations obtained after treatment start are used in a posteriori TDM [15]. Following the detection of non-optimal drug concentrations, different procedures for dose adjustments are possible: in the simplest case, oncologists will use the drug label, dosing algorithms or nomograms to determine a new dose [27]. Although simple, this approach requires to abide with the scheduled blood sampling times and is unsuitable if the patient is not represented by the population on which drug label or dosing algorithm have been developed on [28].

Another a posteriori approach involves the collection of 4 8 blood samples within a dosing interval and the subsequent calculation of the area under the concentration-time curve (AUC) [11]. Based on the calculated AUC, individual PK parameters can be obtained and used for PK calculations to 
determine a more suitable dose. However, dense blood sampling is rarely feasible in clinical practice [29].

Population PK (nonlinear mixed-effects) modelling and simulation [30] can aid in optimising TDM in multiple ways: first, PK information from the population can be incorporated into model parameters during model development. Use of this information allows to refrain from dense blood sampling in model-informed precision dosing (MIPD) and often few samples are enough to obtain sufficiently precise individual PK estimates [26]. Second, sampling at fixed time points is no longer necessary and can also be performed prior to steadystate attainment [26]. As long as actual sampling times are documented, samples from virtually every time point can be used for PK analyses in MIPD [26]. Still, there are more and less informative sampling time points. Optimal design, another part of the model-informed dose individualisation process, can aid in systematically determining the most informative sampling time point(s) within a given time frame [31]. Finally, Bayesian TDM in MIPD combines model-informed TDM with the ability to learn and subsequently forecast drug concentrations at various possible dosing regimens. Similarly to traditional population PK, the Bayesian approach uses information from the population to estimate the most likely PK parameter values for a given drug and population [32]. If specific patient characteristics influence one or more of the PK processes, this information can already be used in an a priori dose selection process. At the beginning of treatment, when no concentration measurements are available, predicted PK parameter values for a specific patient will be identical with the population estimates [29]. As measured drug concentrations become available, they are used to refine the patient's predicted PK parameter values. The more patient-individual information (i.e. drug concentrations) is available, the more weight is set on this information in the parameter estimation process and the more individual parameter estimates will be allowed to deviate from the population estimates [29]. Moreover, Bayesian TDM can account for IOV that is lower than the safe and effective variability [33] and still predict future doses based on at least two sampling occasions [34, 35]. A disadvantage of Bayesian TDM is the high shrinkage of predicted individual PK parameters if only a single PK sample is available: when the population outweighs the individual information, individual information on the patient will get lost as the empirical Bayes estimates shrinks to the typical population parameters [29]. Moreover, applying Bayesian TDM requires special knowledge, can be time intensive and thus difficult to implement in clinical practice.

Sampling minimum plasma concentrations at steady-state $\left(\mathrm{C}_{\text {min,ss }}\right)$ is often performed in clinical practice and, if done correctly, the currently most precise approach as it avoids shrinkage of individual information to the population mean. However, it requires precise information about the patient's dosing schedule and good coordination between patient and treatment team. An easy and time-efficient way to circumvent the need to sample at $\mathrm{C}_{\min , \mathrm{SS}}$ is to account for the difference between the time of minimum concentrations and time of measurement and extrapolate based on the time after last dose and the terminal half-life of the drug. In this method, based on an algorithm described and validated for imatinib [10], samples can be taken at random time points in the elimination phase of the drug and the corresponding $\mathrm{C}_{\mathrm{min}, \mathrm{Ss}}$ can be calculated using Eq. (1).

$C_{\text {min, SS }}=C_{\text {measured }} * 0.5^{\frac{\text { Dosing Interval [h]-Time after last dose }[\mathrm{h}]}{\text { Half-life }[\mathrm{h}]}}$

Of note, this method assumes that $\mathrm{C}_{\text {measured }}$ is sampled in the terminal phase of a monoexponential decline. For drugs with a nonlinear clearance or a short half-life (i.e. dasatinib, axitinib), an alternative method has to be used. For example, the $\mathrm{C}_{\mathrm{min}, \mathrm{SS}}$ can also be estimated based on a randomly taken concentration measurement $\left(\mathrm{C}_{\text {measured }}\right)$ and a simulated typical concentration-time curve, using an existing population PK model. Based on the ratio of the measured concentration at $t_{\text {measured }}$ with the concentration in the simulated PK profile, the corresponding $\mathrm{C}_{\min , \mathrm{SS}}$ in this patient can be estimated [36].

\section{Therapeutic drug monitoring for oral targeted antineoplastic drugs}

Several OAD characteristics suggest individualised dosing:

1 OADs show highly variable drug exposure, caused by IIV in absorption, distribution, metabolism and excretion (ADME). Oral bioavailability (BA) differs between and within agents (i.e. $14-34 \%$ in dasatinib and $98 \%$ in imatinib) and depends on drug formulation [37], absorption, first-pass hepatic metabolism and food intake. Moreover, almost all OADs are metabolised by monooxygenases of the Cytochrome P450 (CYP) family [3]. Up to 20-fold variability in expression and activity of CYP3A4 has been reported, and polymorphisms in the isoenzymes CYP2D6, CYP2C9 and CYP2C19 additionally contribute to the variable metabolic activity [3]. The activity of CYP enzymes may be additionally influenced by concomitant administration of CYP inducers/inhibitors, environmental factors, smoking and food intake $[3,38]$. Polymorphic transporters are also involved in the excretion of many agents (i.e. axitinib, dasatinib and sorafenib) [3].

2 Efficacy is challenging to assess during OAD treatment, as benefits in clinical outcome parameters such as overall survival (OS) and progression-free survival (PFS) take long until evaluable. Objective response rates using CT scans can be assessed earlier and for a few malignancies, reliable biomarkers are available (i.e. prostate specific antigen for prostate cancer or complete cytogenic response 
(CCyR) for chronic myeloid leukaemia (CML)). Furthermore, advances in PKPD modelling allow to use tumour dynamics in exposure-response analyses [39]. If an exposure-response relationship has been established, achieving target concentrations can serve as a proxy for achieving beneficial outcomes.

As disease progression can be fatal in oncology, treatment at an exposure above the efficacy threshold should be assured from the start of treatment or at least achieved as soon as possible, while individual patient toxicity should be monitored carefully. Furthermore, dose increases should only be implemented in case of acceptable toxicity and patients with low exposure and considerable toxicity should be switched to another treatment option.

Of note, while TDM might be crucial for agents with a narrow therapeutic window (i.e. pazopanib, sunitinib), it might be less relevant for agents with a wider therapeutic window (i.e. erlotinib, osimertinib).

Considering the high costs of OADs, cost-neutral PK-guided dose interventions to increase exposure, i.e. concomitant intake with food [40-42], split intake moments [43] or boosting (i.e. with a CYP3A4 inhibitor), should be considered before conventional dose increments, particularly in countries with poor healthcare systems.

For some agents, TDM has already proven feasible [44]. Strong evidence exists for imatinib in CML [18, 45] and gastrointestinal stromal tumours (GIST) [46]. Additional compounds for which TDM was feasible in prospective studies are sunitinib [47], pazopanib [48], tamoxifen [20] and abiraterone [40]. For other agents, i.e. alectinib [49], axitinib, crizotinib [49], trametinib [50] and vemurafenib [51-54], a PK target associated with either efficacy or toxicity has been established, but not yet evaluated in prospective clinical studies [13]. Lastly, no information about the value of TDM is available for some compounds. Most of these are new, and exposure-response relationships have not been established yet. For those drugs, we suggest to target the mean/median exposure as proxy for a PK target, as previously established PK targets amounted to $85 \%( \pm 19 \%)[14]$ and $82 \%( \pm 17 \%)$ [13] of the mean population exposures in AHDs and KIs, respectively. This is already applied in the DPOG-TDM study [55] and similar approaches are suggested by the FDA for special populations [56-58]. In the DPOG-TDM study [55], the feasibility, tolerability and efficacy of TDM for 23 different OADs is currently being evaluated (www.trialregister.nl; NL6695)) and preliminary results are promising [59].

The rapid improvement in OAD treatment together with the continuous development of new compounds poses a challenge for the timely establishment of viable TDM targets. While exposure-safety relationships are determined early during drug development, observing exposure-response relationships requires extensive time. Accordingly, there is often a discrepancy between the level of viability of proposed PK/ PD targets and the clinical relevance of a compound. For example, while imatinib was approved in 2001 [1], the exposure-response relationships in CML and GIST became publicly available in 2008 [45] and 2009 [46], respectively. Sunitinib was first approved in 2006, but the exposureresponse relationship was published in 2010 [60]. Likewise, pazopanib was approved by the EMA in 2010 and the exposure-response relationship was first described in 2014 [61].

In the following section, we explore the potential of TDMguided dosing to optimise OAD treatment. For each drug, we searched PubMed and Google Scholar using the terms 'pharmacokinetics', 'exposure response', 'exposure efficacy', 'exposure safety', 'exposure toxicity', 'therapeutic drug monitoring' and 'TDM' together with the respective drug name. Additionally, we reviewed the respective EMA European Public Assessment Reports and the FDA Clinical Pharmacology and Biopharmaceutics Reviews. In Supplementary Tables 1a-c, KIs, AHDs and other OADs are summarised together with their molecular target(s), therapeutic indication and date of first approval. Table 1 presents current evidence for TDM-guided dosing of OADs. In this table, each drug is classified according to the level of evidence currently available for TDM. If there is an established exposureresponse relationship and a PK target, TDM is considered potentially useful. If additionally, a feasibility study has been performed, TDM is recommended. If on top of that, randomised, prospective studies demonstrated a positive effect of TDM, it is strongly recommended. If there is no evidence for an exposure-response relationship, TDM is considered exploratory. If there are minimal data on the PK of a drug, there are more useful targets than plasma concentration or there is evidence that TDM is not useful, it is not recommended. Compounds with the highest clinical relevance as monotherapy and for which TDM is classified as recommended are discussed below.

\section{Abiraterone}

In an observational study in 61 metastatic castration-resistant prostate cancer patients, $\mathrm{C}_{\min } \geq 8.4 \mathrm{ng} / \mathrm{mL}$ were associated with a significantly longer PFS compared to $\mathrm{C}_{\min }<8.4 \mathrm{ng} /$ mL (PFS 7.4 vs 12.2 months, $p=0.044$ ) [62]. This threshold was later confirmed in a real-world patient cohort $(n=62$, PFS 6.1 vs. 16.9 months, $p=0.033$ ) [63]. Yet, at the standard dose of $1000 \mathrm{mg}$ once daily (QD), $35 \%$ and $42 \%$ of patients, respectively, did not reach this target $[62,63]$. A prospective study $(n=32)$ demonstrated that 20 patients $(63 \%)$ had at least one $\mathrm{C}_{\min }<8.4 \mathrm{ng} / \mathrm{mL}$ with standard care [40]; however, when a light meal or snack was concomitantly taken with abiraterone, adequate exposure in 28 patients $(87.5 \%)$ without 
additional toxicities was achieved [40]. Thus, TDM of abiraterone and concomitant food intake as a cost-neutral PK-guided intervention to reach $\mathrm{C}_{\min }>8.4 \mathrm{ng} / \mathrm{mL}$ has proven feasible. Given the absence of an exposure-toxicity relationship, a pragmatic option could be to include concomitant food intake in the drug label.

\section{Everolimus}

In a meta-analysis, it has been reported that a two-fold increase in $\mathrm{C}_{\min }$ was linked to an increased reduction in tumour size and $\mathrm{C}_{\mathrm{SS}, \min } \geq 10 \mathrm{ng} / \mathrm{mL}$ could be used as a cut-off value [64]. At the same time, $C_{\mathrm{SS}, \min }>26.3 \mathrm{ng} / \mathrm{mL}$ have been associated with a 4-fold increased risk of toxicity compared to $\mathrm{C}_{\mathrm{SS} \text {,min }}<26.3 \mathrm{ng} / \mathrm{mL}$ [21]. As the occurrence of AEs seemed to be associated with high maximum concentrations $\left(\mathrm{C}_{\max }\right)$ [65], Verheijen et al. investigated the potential of alternative dosing to reduce $\mathrm{C}_{\max }$-related AEs while maintaining therapeutic $\mathrm{C}_{\mathrm{SS} \text {,min. }}$. In a crossover study in 11 patients, administering $5 \mathrm{mg}$ twice daily (BID) instead of $10 \mathrm{mg}$ QD significantly reduced everolimus $\mathrm{C}_{\max }$ while $\mathrm{C}_{\mathrm{SS} \text {, min }}$ increased from 9.6 to $13.7 \mathrm{ng} / \mathrm{mL}$ [65]. Given the established exposureresponse and exposure-toxicity relationships, we propose to combine $5 \mathrm{mg}$ BID dosing with TDM to target a therapeutic window of $\mathrm{C}_{\mathrm{SS} \text {, min }} \geq 10 \mathrm{ng} / \mathrm{mL}$ and $<26.3 \mathrm{ng} / \mathrm{mL}$. The developed population PK model by Combes et al. [66] could serve as a starting point in a MIPD framework.

\section{Imatinib}

Higher frequencies of CCyR and major molecular response (MMR) have been reported in CML patients with high imatinib $\mathrm{C}_{\min , \mathrm{SS}}[45,67]$. Current evidence supports the use of a
$\mathrm{C}_{\min , \mathrm{SS}} \geq 1000 \mathrm{ng} / \mathrm{mL}$ as $\mathrm{PK}$ target to achieve improved CCyR and MMR in CML [68]. Imatinib $\mathrm{C}_{\text {min,Ss }}>3000 \mathrm{ng} /$ $\mathrm{mL}$ have been associated with higher rates of AEs [67]. Therefore, a therapeutic window of $1000 \leq \mathrm{C}_{\min , \mathrm{SS}}<$ $3000 \mathrm{ng} / \mathrm{mL}$ seems reasonable [68]. In gastrointestinal stromal tumours (GIST), one study determined a longer time to disease progression in patients $(n=73)$ with $\mathrm{C}_{\mathrm{SS}, \min } \geq 1100 \mathrm{ng} /$ $\mathrm{mL}$ [46]. In another study, a significantly longer PFS was found in patients with $\mathrm{C}_{\mathrm{min}, \mathrm{SS}} \geq 760 \mathrm{ng} / \mathrm{mL}$ compared to patients with $\mathrm{C}_{\mathrm{min}, \mathrm{Ss}}<760 \mathrm{ng} / \mathrm{mL}$ (PFS not reached vs. 56 months, respectively), although this patient population was not representative of routine clinical practice [69]. The feasibility of TDM-guided dosing to achieve imatinib $\mathrm{C}_{\min , \mathrm{SS}}$ of $750-1500 \mathrm{ng} / \mathrm{mL}$ has been proven in a prospective randomised controlled trial [18], and several population PK models [70-72] are available for use in MIPD of imatinib. As the fraction of patients reaching durable $\mathrm{C}_{\mathrm{min}, \mathrm{SS}} \geq 1000 \mathrm{ng} / \mathrm{mL}$ has been reported to be as low as $33.3 \%$ [73], individualised imatinib dosing is highly relevant. As imatinib $\mathrm{C}_{\min }$ have been reported to decrease during the first 3 months of treatment [74], it is important to keep measuring imatinib $\mathrm{C}_{\mathrm{min}, \mathrm{SS}}$ during treatment and after dose adjustments.

\section{Pazopanib}

An association of $\mathrm{C}_{\mathrm{SS}, \min } \geq 20.5 \mathrm{mg} / \mathrm{L}$ with improved PFS (19.6 vs. 52.0 weeks, $p=0.004$ ) and tumour shrinkage was found in a retrospective analysis in 177 patients with advanced renal cell carcinoma (RCC) [61]. This efficacy threshold was later validated in the adjuvant setting [75] and in a real-life patient cohort [76]. However, 16-20\% [61, 76] of patients do not reach this threshold and are thus at risk of decreased efficacy. In a prospective feasibility study of individualised

Table 1 Evidence for TDM for targeted oral antineoplastic drugs

\begin{tabular}{cll}
\hline Evidence level & Recommendation & Description \\
\hline 1 & Strongly recommended & $\begin{array}{l}\text { Randomised, prospective studies demonstrated positive effect of } \\
\text { routine TDM with regards to efficacy and/or safety. } \\
\text { There is an established exposure-response relationship using standard } \\
\text { dosage from retrospective studies, a target is established and a } \\
\text { feasibility study has been performed. }\end{array}$ \\
\hline 2 & Recommended & $\begin{array}{l}\text { An exposure-response or exposure-safety relationship using standard } \\
\text { dosage has been identified and a potential target has been reported. }\end{array}$ \\
\hline 4 & Explontially useful & $\begin{array}{l}\text { An exposure-response or exposure-safety relationship using standard } \\
\text { dosage has been identified but no target has been reported }\end{array}$ \\
& & $\begin{array}{l}\text { No exposure-response or exposure-safety relationship using } \\
\text { standard dosage has been identified and/or } \\
\text { - there is very few data on pharmacokinetics of the drug; }\end{array}$ \\
& Not recommended & $\begin{array}{l}\text { - there are more useful targets than plasma concentration (PD); } \\
\text { - there is evidence that TDM is not useful }\end{array}$ \\
\hline
\end{tabular}




\begin{tabular}{|c|c|c|c|c|c|c|c|c|}
\hline Substance & $\begin{array}{l}\text { Evidence } \\
\text { level for } \\
\text { TDM }\end{array}$ & $\begin{array}{l}\text { Proposed } \\
\text { TDM } \\
\text { target } \\
{[\mathrm{ng} / \mathrm{mL}]}\end{array}$ & $\begin{array}{l}\text { Patients } \\
\text { below TDM } \\
\text { target at } \\
\text { standard } \\
\text { dose }(\%)\end{array}$ & $\begin{array}{l}\text { Reported } \\
\text { mean/median } \\
\text { Css,min at } \\
\text { standard dose } \\
{[\mathrm{ng} / \mathrm{mL}]\left(\mathrm{IIV}^{\mathrm{a}}\right)}\end{array}$ & $\begin{array}{l}\text { Exposure-response } \\
\text { relationship } \\
\text { [yes/no] } \\
\text { (associated } \\
\text { parameter(s)) }\end{array}$ & $\begin{array}{l}\text { Exposure-safety } \\
\text { relationship } \\
\text { [yes/no] } \\
\text { (associated } \\
\text { parameter(s)) }\end{array}$ & $\begin{array}{l}\text { NLME } \\
\text { model } \\
\text { available }\end{array}$ & Comments \\
\hline Abiraterone & Recommended & $\begin{array}{l}\mathrm{C}_{\mathrm{SS}, \min } \geq \\
8.4 \\
{[62,63]}\end{array}$ & $\begin{array}{l}35[62]- \\
42[63]\end{array}$ & $9.3(70 \%)[63]$ & Yes (PFS) $[62,63]$ & $\mathrm{No}[63]$ & Yes[99] & $\begin{array}{l}\text { TDM with food intervention } \\
\text { has been proven feasible and } \\
\text { cost-efficient }[40,100]\end{array}$ \\
\hline Axitinib & Recommended & $\begin{array}{l}\mathrm{AUC} \geq \\
300^{\mathrm{b}}[101] \\
\mathrm{C}_{\mathrm{SS}, \min } \\
\geq 5[102]\end{array}$ & $38[101]$ & $\begin{array}{l}\text { AUC: } 367^{b} \\
(77 \%)[103]\end{array}$ & $\begin{array}{l}\text { Yes (PFS, PR, } \\
\text { OS)[101,102] }\end{array}$ & $\begin{array}{l}\text { Yes (hypertension, } \\
\text { proteinuria, fatigue, } \\
\text { diarrhoea)[104] }\end{array}$ & Yes[101] & $\begin{array}{l}\text { - Diastolic blood pressure (dBP) } \\
\text { has additionally been related to } \\
\text { efficacy[105] } \\
\text { - A placebo-controlled } \\
\text { randomised dose titration trial } \\
\text { was successfully } \\
\text { performed[106] } \\
\text { - An integrated approach using } \\
\text { both PK and dBP might be the } \\
\text { most promising option } \\
\text { - Exposure-response relationship } \\
\text { only evaluated for axitinib } \\
\text { monotherapy but not for } \\
\text { combination therapy with an } \\
\text { immune checkpoint inhibitor }\end{array}$ \\
\hline Everolimus & Recommended & $\begin{array}{l}C_{S S, \min } \geq 10 \\
{[64]}\end{array}$ & $37[64]$ & $\begin{array}{l}15.65 \\
(90 \% \text { CI: } 14.79- \\
16.55)[64]\end{array}$ & Yes (PFS) $[64,107]$ & $\begin{array}{l}\text { Yes (pulmonary } \\
\text { events, } \\
\text { stomatitis) }[64,108]\end{array}$ & Yes[109] & $\begin{array}{l}\text { - } 5 \text { mg BID instead of } 10 \mathrm{mg} \text { QD } \\
\text { decreases } \mathrm{C}_{\max } \text { (potentially } \\
\text { decreased risk for toxicity)[65] } \\
\text { - PK-guided dosing has been } \\
\text { proven feasible[110] } \\
\text { - TDM-guided everolimus dosing } \\
\text { already SoC in transplantation } \\
\text { medicine[111] }\end{array}$ \\
\hline Substance & $\begin{array}{l}\text { Evidence } \\
\text { level for } \\
\text { TDM }\end{array}$ & $\begin{array}{l}\text { Proposed } \\
\text { TDM } \\
\text { target } \\
{[\mathrm{ng} / \mathrm{mL}]}\end{array}$ & $\begin{array}{l}\text { Patients } \\
\text { below TDM } \\
\text { target at } \\
\text { standard } \\
\text { dose (\%) } \\
\end{array}$ & $\begin{array}{l}\text { Reported } \\
\text { mean/median } \\
\text { Css,min at } \\
\text { standard dose } \\
{[\mathrm{ng} / \mathrm{mL}]\left(\mathrm{IIV}^{\mathrm{a}}\right)} \\
\end{array}$ & $\begin{array}{l}\text { Exposure-response } \\
\text { relationship } \\
\text { [yes/no] } \\
\text { (associated } \\
\text { parameter(s)) } \\
\end{array}$ & $\begin{array}{l}\text { Exposure-safety } \\
\text { relationship } \\
\text { [yes/no] } \\
\text { (associated } \\
\text { parameter(s)) }\end{array}$ & $\begin{array}{l}\text { NLME } \\
\text { model } \\
\text { available }\end{array}$ & Comments \\
\hline Gefitinib & Recommended & $\begin{array}{l}C_{S S, \min } \geq \\
200[4]\end{array}$ & $30[4]$ & $266(41 \%)[4]$ & Yes (OS)[4] & $\begin{array}{l}\text { Yes (skin toxicity, } \\
\text { diarrhoea, hepato- } \\
\text { toxicity) }[4,112]\end{array}$ & No & $\begin{array}{l}\text { Treatment at increased doses } \\
\text { has been proven feasible[113] } \\
\text { Gefitinib has lost most of its } \\
\text { relevance in the treatment of } \\
\text { EGFR mutated lung cancer }\end{array}$ \\
\hline Imatinib & Recommended & $\begin{array}{l}\text { CML: } \\
\text { C }_{S S, \min } \\
\geq 1000 \\
{[45,67,114} \\
] \\
\text { GIST: } \\
\text { C }_{S S, \min } \geq \\
1100[46]\end{array}$ & $73[115]$ & $926(52 \%)$ [115] & $\begin{array}{l}\text { Yes (MMR, CcyR) } \\
{[45,67,114]} \\
\text { Yes (TTP) }[46,69]\end{array}$ & $\begin{array}{l}\text { Yes (neutropenia, } \\
\text { rash, diarrhoea, } \\
\text { arthralgia, oedema) } \\
{[67,116,117]}\end{array}$ & Yes[70] & $\begin{array}{l}\text { Feasibility of TDM has been } \\
\text { proven in a cohort study[44] } \\
\text { and a RCT[18] } \\
\text { - An alternative threshold of } \\
760 \mathrm{ng} / \mathrm{mL} \text { has been proposed } \\
\text { for GIST [69] }\end{array}$ \\
\hline Pazopanib & Recommended & $\begin{array}{l}\mathrm{C}_{\mathrm{SS}, \min } \geq \\
20500 \\
{[61,75,76]}\end{array}$ & $\begin{array}{l}16[76]- \\
20[61]\end{array}$ & $\begin{array}{l}28100 \\
(40 \%)[76]\end{array}$ & $\begin{array}{l}\text { Yes (PFS) } \\
{[61,75,76]}\end{array}$ & $\begin{array}{l}\text { Yes (fatigue, } \\
\text { anorexia, } \\
\text { hypertension) [77] }\end{array}$ & Yes[78] & $\begin{array}{l}\text { - PK-guided dosing has been } \\
\text { proven feasible[48] } \\
\text { - } 400 \mathrm{mg} \text { BID instead of } 800 \mathrm{mg} \\
\text { QD as cost-neutral strategy to } \\
\text { increase exposure[43] } \\
\text { - Concomitant intake with food } \\
\text { as cost-neutral strategy to } \\
\text { increase exposure[42,118] }\end{array}$ \\
\hline Sunitinib & Recommended & $\begin{array}{l}\text { Inter- } \\
\text { mittent } \\
\text { dosing: } \\
\mathrm{C}_{\mathrm{SS}, \min } \geq \\
50^{\mathrm{c}}[60] \\
\text { Continuous } \\
\text { dosing: }\end{array}$ & $\begin{array}{l}49[115]- \\
52[119]\end{array}$ & $51.6(39 \%)[115]$ & Yes (TTP, OS)[60] & $\begin{array}{l}\text { Yes (hypertension, } \\
\text { fatigue, anorexia, } \\
\text { myelosuppression, } \\
\text { HFSR, altered taste, } \\
\text { mucositis) } \\
{[79,80,120,121]}\end{array}$ & Yes[81] & $\begin{array}{l}\text { PK-guided dosing has been } \\
\text { proven feasible[119] }\end{array}$ \\
\hline
\end{tabular}




\begin{tabular}{|c|c|c|c|c|c|c|c|c|}
\hline Substance & $\begin{array}{l}\text { Evidence } \\
\text { level for } \\
\text { TDM }\end{array}$ & $\begin{array}{l}\text { Proposed } \\
\text { TDM } \\
\text { target } \\
{[\mathrm{ng} / \mathrm{mL}]}\end{array}$ & $\begin{array}{l}\text { Patients } \\
\text { below TDM } \\
\text { target at } \\
\text { standard } \\
\text { dose (\%) } \\
\end{array}$ & $\begin{array}{l}\text { Reported } \\
\text { mean/median } \\
\text { CSs,min at } \\
\text { standard dose } \\
{[\mathrm{ng} / \mathrm{mL}](\mathrm{IIV})} \\
\end{array}$ & $\begin{array}{l}\text { Exposure-response } \\
\text { relationship } \\
\text { [yes/no] } \\
\text { (associated } \\
\text { parameter(s)) } \\
\end{array}$ & $\begin{array}{l}\text { Exposure-safety } \\
\text { relationship } \\
\text { [yes/no] } \\
\text { (associated } \\
\text { parameter(s)) }\end{array}$ & $\begin{array}{l}\text { NLME } \\
\text { model } \\
\text { available }\end{array}$ & Comments \\
\hline & & $\begin{array}{l}\mathrm{C}_{\mathrm{SS}, \min } \geq \\
37.5^{\mathrm{c}}[12]\end{array}$ & & & & & & \\
\hline Tamoxifen & Recommended & $\begin{array}{l}\mathrm{C}_{\mathrm{Ss}} \geq 5.97^{\mathrm{d}} \\
{[83]}\end{array}$ & $20[83]$ & $\begin{array}{l}9.72^{\mathrm{d}} \\
<1.73-30.8>\mathrm{d} \\
{[122]}\end{array}$ & Yes (RR)[83] & No[89] & Yes[92] & $\begin{array}{l}\text { TDM-guided dosing has been } \\
\text { proven feasible[20] } \\
\text { - Genotype-guided dose } \\
\text { escalations have been proven } \\
\text { safe[87-89] }\end{array}$ \\
\hline Trametinib & Recommended & $\begin{array}{l}\mathrm{C}_{\mathrm{SS}, \min } \geq \\
10.6[50]\end{array}$ & $27[123]$ & $\begin{array}{l}12.1<6- \\
34>[124]\end{array}$ & Yes (PFS)[50] & No[124] & Yes[50] & $\begin{array}{l}\text { Exposure-response relationship } \\
\text { only evaluated for trametinib } \\
\text { monotherapy but not for } \\
\text { combination therapy with } \\
\text { dabrafenib }\end{array}$ \\
\hline Alectinib & $\begin{array}{l}\text { Potentially } \\
\text { useful }\end{array}$ & $\begin{array}{l}\mathrm{C}_{\text {Ss,min }} \geq \\
435 \\
{[49,125]}\end{array}$ & $33[125]$ & $572(48 \%)[126]$ & $\begin{array}{l}\text { Yes (tumour size, } \\
\text { PFS) }[49,125]\end{array}$ & $\mathrm{No}[127]$ & Yes[126] & \\
\hline Crizotinib & $\begin{array}{l}\text { Potentially } \\
\text { useful }\end{array}$ & $\begin{array}{l}\mathrm{C}_{\mathrm{SS}, \min } \geq \\
235 \\
{[49,128]}\end{array}$ & $48[49]$ & $244(45 \%)$ [49] & $\begin{array}{l}\text { Yes (PFS, ORR) } \\
{[49,128]}\end{array}$ & $\begin{array}{l}\text { Yes (neutropenia, } \\
\text { AST } \\
\text { elevation)[129] }\end{array}$ & Yes[130] & \\
\hline Erlotinib & $\begin{array}{l}\text { Potentially } \\
\text { useful }\end{array}$ & $\begin{array}{l}\mathrm{C}_{\mathrm{SS}, \min }> \\
500[131]\end{array}$ & $11[115]$ & $\begin{array}{l}1011 \\
(69 \%)[115]\end{array}$ & $\begin{array}{l}\text { Yes (preclinical } \\
\text { efficacy, PFS, } \\
\text { OS)[131,132] }\end{array}$ & $\begin{array}{l}\text { Yes (skin toxicity) } \\
{[133,134]}\end{array}$ & Yes[135] & \\
\hline Substance & $\begin{array}{l}\text { Evidence } \\
\text { level for } \\
\text { TDM }\end{array}$ & $\begin{array}{l}\text { Proposed } \\
\text { TDM } \\
\text { target } \\
{[\mathrm{ng} / \mathrm{mL}]}\end{array}$ & $\begin{array}{l}\text { Patients } \\
\text { below TDM } \\
\text { target at } \\
\text { standard } \\
\text { dose }(\%)\end{array}$ & $\begin{array}{l}\text { Reported } \\
\text { mean/median } \\
\text { Css,min at } \\
\text { standard dose } \\
{[\mathrm{ng} / \mathrm{mL}]\left(\mathrm{IIV}^{\mathrm{a}}\right)}\end{array}$ & $\begin{array}{l}\text { Exposure-response } \\
\text { relationship } \\
\text { [yes/no] } \\
\text { (associated } \\
\text { parameter(s)) }\end{array}$ & $\begin{array}{l}\text { Exposure-safety } \\
\text { relationship } \\
\text { [yes/no] } \\
\text { (associated } \\
\text { parameter(s)) }\end{array}$ & $\begin{array}{l}\text { NLME } \\
\text { model } \\
\text { available }\end{array}$ & Comments \\
\hline Gilteritinib & $\begin{array}{l}\text { Potentially } \\
\text { useful }\end{array}$ & $\begin{array}{l}\mathrm{C}_{\mathrm{SS}, \min }>10 \\
0[136]\end{array}$ & $0.6[136]$ & $\begin{array}{l}456(\mathrm{NA}) \\
{[136]}\end{array}$ & Yes (CR)[136] & $\begin{array}{l}\text { Yes (CK, AST, } \\
\text { ALT, ALB)[136] }\end{array}$ & Yes[136] & \\
\hline Letrozole & $\begin{array}{l}\text { Potentially } \\
\text { useful }\end{array}$ & $\begin{array}{l}\mathrm{C}_{\mathrm{SS}, \min }> \\
85.6[137]\end{array}$ & NA & $\begin{array}{l}88.4 \\
<0-349.2>[138]\end{array}$ & Yes (TTP)[137] & Not reported & Yes[139] & \\
\hline Nilotinib & $\begin{array}{l}\text { Potentially } \\
\text { useful }\end{array}$ & $\begin{array}{l}\mathrm{C}_{S S, \min } \\
\geq 469[140]\end{array}$ & $25[140]$ & $\begin{array}{l}1123(64 \%)(300 \\
\mathrm{mg} \text { BID })[141] \\
1239(52 \%)(400 \\
\mathrm{mg} \mathrm{BID})[141]\end{array}$ & $\begin{array}{l}\text { Yes (TTP, trend for } \\
\text { MMR) }[140,141]\end{array}$ & $\begin{array}{l}\text { Yes (bilirubin and } \\
\text { liver enzyme } \\
\text { elevations) [140- } \\
142]\end{array}$ & Yes[140] & $\begin{array}{l}\text { Feasibility of TDM has been } \\
\text { reported in a case study [143] } \\
\text { Dose selection with respect to } \\
\text { UGT1A1 genotype could } \\
\text { prevent elevation of bilirubin } \\
\text { levels[142] }\end{array}$ \\
\hline Vemurafenib & $\begin{array}{l}\text { Potentially } \\
\text { useful }\end{array}$ & $\begin{array}{l}\mathrm{C}_{\mathrm{SS}, \min } \geq 420 \\
00[51-53]\end{array}$ & $\begin{array}{l}39[144]- \\
52[145]\end{array}$ & $\begin{array}{l}61000(41 \%)[14 \\
4]\end{array}$ & $\begin{array}{l}\text { Yes (PFS, OS) } \\
{[51-54]}\end{array}$ & $\begin{array}{l}\text { Yes }(\mathrm{QTc} \\
\text { prolongation, } \\
\text { rash) }[53,146]\end{array}$ & Yes[147] & $\begin{array}{l}\text { - An alternative threshold of } \\
\mathrm{C}_{\mathrm{SS}, \min }>50 \mathrm{mg} / \mathrm{L} \text { has been } \\
\text { proposed [54] }\end{array}$ \\
\hline Abemaciclib & Exploratory & NA & NA & $181(47 \%)[148]$ & $\begin{array}{l}\text { Yes (tumour } \\
\text { shrinkage, BOR, } \\
\text { PFS) }[148]\end{array}$ & $\begin{array}{l}\text { Yes (neutropenia) } \\
{[148]}\end{array}$ & Yes[149] & \\
\hline Afatinib & Exploratory & NA & NA & $\begin{array}{l}\text { NA } \\
<14.4- \\
27.4>[150]\end{array}$ & $\mathrm{No}[150]$ & $\begin{array}{l}\text { Yes (diarrhoea, } \\
\text { anorexia)[150-152] }\end{array}$ & Yes[153] & $\begin{array}{l}\text { - Daily doses }<20 \mathrm{mg} \text { have } \\
\text { been associated with a } \\
\text { significant shorter PFS[152] }\end{array}$ \\
\hline Alpelisib & Exploratory & NA & NA & $\begin{array}{l}\text { AUC: } 33224 \\
(21 \%)^{b}[154]\end{array}$ & $\begin{array}{l}\text { Inconclusive (trend } \\
\text { for PFS) }[154]\end{array}$ & $\begin{array}{l}\text { Yes (hyper- } \\
\text { glycemia) [154] }\end{array}$ & $\begin{array}{l}\text { Yes[155 } \\
]\end{array}$ & \\
\hline Anastrozole & Exploratory & NA & NA & $\begin{array}{l}33.2 \\
<0.0-98.8> \\
{[156]}\end{array}$ & $\begin{array}{l}\text { Yes (oestradiol } \\
\text { suppression) } \\
{[156]}\end{array}$ & Not reported & NA & $\begin{array}{l}\text { Relationship of anastrozole } \\
\text { CSs,min with both oestrogen }_{\text {levels and efficacy endpoints }} \\
\text { should be further } \\
\text { investigated[14] }\end{array}$ \\
\hline
\end{tabular}




\begin{tabular}{|c|c|c|c|c|c|c|c|c|}
\hline Substance & $\begin{array}{l}\text { Evidence } \\
\text { level for } \\
\text { TDM }\end{array}$ & $\begin{array}{l}\text { Proposed } \\
\text { TDM } \\
\text { target } \\
{[\mathrm{ng} / \mathrm{mL}]}\end{array}$ & $\begin{array}{l}\text { Patients } \\
\text { below TDM } \\
\text { target at } \\
\text { standard } \\
\text { dose (\%) } \\
\end{array}$ & $\begin{array}{l}\text { Reported } \\
\text { mean/median } \\
\text { CSs,min at } \\
\text { standard dose } \\
{[\mathrm{ng} / \mathrm{mL}]\left(\mathrm{IIV}^{\mathrm{a}}\right)} \\
\end{array}$ & $\begin{array}{l}\text { Exposure-response } \\
\text { relationship } \\
\text { [yes/no] } \\
\text { (associated } \\
\text { parameter(s)) } \\
\end{array}$ & $\begin{array}{l}\text { Exposure-safety } \\
\text { relationship } \\
\text { [yes/no] } \\
\text { (associated } \\
\text { parameter(s)) }\end{array}$ & $\begin{array}{l}\text { NLME } \\
\text { model } \\
\text { available }\end{array}$ & Comments \\
\hline & & & & & & & & $\begin{array}{l}\text { A target of } \mathrm{C}_{\mathrm{SS}, \min } \geq 34.2 \\
\mathrm{ng} / \mathrm{mL} \text { has been proposed } \\
{[156]}\end{array}$ \\
\hline Apalutamide & Exploratory & NA & NA & $\begin{array}{l}3700 \\
(32 \%)[157]\end{array}$ & No[157] & $\begin{array}{l}\text { Yes (fatigue, fall, } \\
\text { rash, weight loss, } \\
\text { arthralgia) [157] }\end{array}$ & Yes[158] & \\
\hline Avapritinib & Exploratory & NA & NA & 593 (NA)[159] & No (ORR)[159] & $\begin{array}{l}\text { Yes (G 3/4 AEs, } \\
\text { cognitive } \\
\text { effects)[159] }\end{array}$ & Yes[159] & $\begin{array}{l}\text { Because of the high response } \\
\text { rate and small sample size, the } \\
\text { results of the exposure- } \\
\text { response analysis should be } \\
\text { interpreted with caution[159] }\end{array}$ \\
\hline Binimetinib & Exploratory & NA & NA & $\begin{array}{l}53.3(49 \%) \\
{[160]}\end{array}$ & Yes (PFS)[161] & $\begin{array}{l}\text { Yes (CK increases, } \\
\text { retinal events)[161] }\end{array}$ & Yes[161] & \\
\hline Bosutinib & Exploratory & NA & NA & $\begin{array}{l}400 \mathrm{mg} \text { QD: } \\
112(73 \%) \\
500 \mathrm{mg} \text { QD: } \\
147(70 \%)[162]\end{array}$ & $\begin{array}{l}\text { Yes (CcyR, MMR, } \\
\text { CHR) }[163,164]\end{array}$ & $\begin{array}{l}\text { Yes (diarrhoea, } \\
\text { rash)[164] }\end{array}$ & Yes[162] & \\
\hline Brigatinib & Exploratory & NA & NA & $\begin{array}{l}520(61 \%) \\
{[165]}\end{array}$ & $\begin{array}{l}\text { Yes (PFS, OS) } \\
{[165]}\end{array}$ & $\begin{array}{l}\text { Yes (diarrhoea, } \\
\text { increased CK, skin } \\
\text { events, } \\
\text { pneumonitis, } \\
\text { pneumonia)[165] }\end{array}$ & Yes[165] & \\
\hline Cabozantinib & Exploratory & NA & NA & $\begin{array}{l}\text { Pred. } \text { Cavg,ss: }_{\text {: }} \\
1125 \text { (NA) } \\
{[166]}\end{array}$ & $\begin{array}{l}\text { Yes (PFS) } \\
{[166,167]}\end{array}$ & $\begin{array}{l}\text { Yes (HFS, fatigue, } \\
\text { diarrhoea, } \\
\text { hypertension)[166] }\end{array}$ & Yes[168] & $\begin{array}{l}\text { - Concomitant intake with food } \\
\text { as cost-neutral PK-guided } \\
\text { intervention [41] }\end{array}$ \\
\hline Ceritinib & Exploratory & NA & NA & $871(47 \%)$ [169] & $\begin{array}{l}\text { Inconclusive (trend } \\
\text { for ORR) }[170]\end{array}$ & $\begin{array}{l}\text { Yes (ALT and AST } \\
\text { elevation, hyper- } \\
\text { glycaemia)[169] }\end{array}$ & Yes[171] & \\
\hline Substance & $\begin{array}{l}\text { Evidence } \\
\text { level for } \\
\text { TDM }\end{array}$ & $\begin{array}{l}\text { Proposed } \\
\text { TDM } \\
\text { target } \\
{[\mathrm{ng} / \mathrm{mL}]}\end{array}$ & $\begin{array}{l}\text { Patients } \\
\text { below TDM } \\
\text { target at } \\
\text { standard } \\
\text { dose }(\%) \\
\end{array}$ & $\begin{array}{l}\text { Reported } \\
\text { mean/median } \\
\text { Css,min at } \\
\text { standard dose } \\
{[\mathrm{ng} / \mathrm{mL}]\left(\mathrm{IIV}^{\mathrm{a}}\right)} \\
\end{array}$ & $\begin{array}{l}\text { Exposure-response } \\
\text { relationship } \\
\text { [yes/no] } \\
\text { (associated } \\
\text { parameter(s)) } \\
\end{array}$ & $\begin{array}{l}\text { Exposure-safety } \\
\text { relationship } \\
\text { [yes/no] } \\
\text { (associated } \\
\text { parameter(s)) }\end{array}$ & $\begin{array}{l}\text { NLME } \\
\text { model } \\
\text { available }\end{array}$ & Comments \\
\hline Dabrafenib & Exploratory & NA & NA & $46.6(84 \%)[172]$ & No[173] & $\begin{array}{l}\text { Yes (AEs requiring } \\
\text { dose } \\
\text { reduction) }[174]\end{array}$ & Yes[172] & $\begin{array}{l}\text { As AUC seems to differ } \\
\text { between dose levels but } C_{S S, m i n} \\
\text { not, AUC might be a more } \\
\text { useful measure of } \\
\text { exposure[172] }\end{array}$ \\
\hline Dacomitinib & Exploratory & NA & NA & $73.1(45 \%)[175]$ & $\begin{array}{l}\text { Inconclusive (trend } \\
\text { for PFS, tumour } \\
\text { shrinkage) [175] }\end{array}$ & $\begin{array}{l}\text { Yes } \\
\text { (rash/dermatitis, } \\
\text { diarrhoea, treatment } \\
\text { alteration) [175] }\end{array}$ & Yes[175] & \\
\hline Dasatinib & Exploratory & NA & NA & $2.61(26 \%)[176]$ & $\begin{array}{l}\text { Yes (McyR, } \\
\text { MR) [176,177] }\end{array}$ & $\begin{array}{l}\text { Yes (pleural } \\
\text { effusions, treatment } \\
\text { alteration) } \\
{[176,178]}\end{array}$ & Yes[179] & \\
\hline Enasidenib & Exploratory & NA & NA & $\begin{array}{l}\mathrm{AUC}_{0-24:} 21515^{\mathrm{b}} \\
(60 \%)[180]\end{array}$ & $\begin{array}{l}\text { Yes (ORR, only for } \\
\text { patients with R140 } \\
\text { mutation) }[180]\end{array}$ & $\begin{array}{l}\text { Yes (bilirubin } \\
\text { elevation, hepatic } \\
\text { safety) }[180]\end{array}$ & Yes[180] & $\begin{array}{l}\text { A trend for an exposure- } \\
\text { response relationship (ORR) } \\
\text { has been reported for patients } \\
\text { with R172 mutation[180] }\end{array}$ \\
\hline Encorafenib & Exploratory & NA & NA & $23(320 \%)[181]$ & $\mathrm{No}[181]$ & $\begin{array}{l}\text { Yes (G 3/4 AEs, } \\
\text { G1+ retinopathy) } \\
{[181]}\end{array}$ & Yes[181] & \\
\hline Erdafitinib & Exploratory & NA & NA & $\begin{array}{l}\text { AUC: } 36608^{b} \\
\text { (NA) [182] }\end{array}$ & $\begin{array}{l}\text { Inconclusive (trend } \\
\text { for PFS) [182] }\end{array}$ & $\begin{array}{l}\text { Yes (nail } \\
\text { disorders)[182] }\end{array}$ & Yes[183] & $\begin{array}{l}\text { Statistically significant } \\
\text { relationships between average } \\
\text { daily serum phosphate } \\
\text { concentrations and both } \\
\text { response and safety have been } \\
\text { reported[182] }\end{array}$ \\
\hline Fedratinib & Exploratory & NA & NA & $\begin{array}{l}\text { AUC: } 33500^{b} \\
(41 \%)[184]\end{array}$ & $\begin{array}{l}\text { Inconclusive (trend } \\
\text { for change in spleen }\end{array}$ & $\begin{array}{l}\text { Yes (anaemia, } \\
\text { thrombocytopenia, }\end{array}$ & Yes[186] & \\
\hline
\end{tabular}




\begin{tabular}{|c|c|c|c|c|c|c|c|c|}
\hline Substance & $\begin{array}{l}\text { Evidence } \\
\text { level for } \\
\text { TDM }\end{array}$ & $\begin{array}{l}\text { Proposed } \\
\text { TDM } \\
\text { target } \\
{[\mathrm{ng} / \mathrm{mL}]}\end{array}$ & $\begin{array}{l}\text { Patients } \\
\text { below TDM } \\
\text { target at } \\
\text { standard } \\
\text { dose (\%) } \\
\end{array}$ & $\begin{array}{l}\text { Reported } \\
\text { mean/median } \\
\text { CSS,min at }_{\text {standard dose }} \\
{[\mathrm{ng} / \mathrm{mL}]\left(\mathrm{IIV}^{\mathrm{a}}\right)} \\
\end{array}$ & $\begin{array}{l}\text { Exposure-response } \\
\text { relationship } \\
\text { [yes/no] } \\
\text { (associated } \\
\text { parameter(s)) } \\
\end{array}$ & $\begin{array}{l}\text { Exposure-safety } \\
\text { relationship } \\
\text { [yes/no] } \\
\text { (associated } \\
\text { parameter(s)) } \\
\end{array}$ & $\begin{array}{l}\text { NLME } \\
\text { model } \\
\text { available }\end{array}$ & Comments \\
\hline & & & & & $\begin{array}{l}\text { volume, STAT3 } \\
\text { phosphorylation) } \\
{[184,185]}\end{array}$ & $\begin{array}{l}\text { diarrhoea, nausea, } \\
\text { vomiting, G3 } \\
\text { AEs) }[184]\end{array}$ & & \\
\hline Glasdegib & Exploratory & NA & NA & $365(81 \%)[187]$ & $\mathrm{No}[187]$ & $\begin{array}{l}\text { Yes (renal toxicity, } \\
\text { dysgeusia, QT } \\
\text { prolongation)[187] }\end{array}$ & Yes[188] & \\
\hline Ibrutinib & Exploratory & NA & NA & AUC: $680^{b}$ [189] & $\begin{array}{l}\text { Yes (BTK } \\
\text { occupancy, clinical } \\
\text { response) [189] }\end{array}$ & $\mathrm{No}[189]$ & Yes[190] & \\
\hline Ivosidenib & Exploratory & NA & NA & $\begin{array}{l}\mathrm{AUC}_{0-24:} \\
117000^{\mathrm{b}} \\
(50 \%)[191]\end{array}$ & $\mathrm{No}[191]$ & $\begin{array}{l}\text { Yes (QTc } \\
\text { prolongation)[191] }\end{array}$ & Yes[191] & \\
\hline Ixazomib & Exploratory & NA & NA & NA & $\begin{array}{l}\text { Yes (clinical } \\
\text { benefit)[192] }\end{array}$ & $\begin{array}{l}\text { Yes (anaemia, } \\
\text { neutropenia, } \\
\text { thrombocytopenia, } \\
\text { diarrhoea, fatigue, } \\
\text { nausea, PN, } \\
\text { rash) }[192,193]\end{array}$ & Yes[194] & \\
\hline Lenvatinib & Exploratory & NA & NA & $\begin{array}{l}43.4(42 \%) \\
(8 \mathrm{mg} \text { QD) } \\
95.6(48 \%) \\
(24 \mathrm{mg} \mathrm{QD})\end{array}$ & $\begin{array}{l}\text { Yes (reduction in } \\
\text { tumour size) } \\
{[195,196]}\end{array}$ & $\begin{array}{l}\text { Yes (treatment } \\
\text { alteration, } \\
\text { hypertension, } \\
\text { proteinuria, nausea, } \\
\text { vomiting, AST, } \\
\text { ALT and bilirubin } \\
\text { elevation) } \\
{[195,197,198]}\end{array}$ & Yes[199] & $\begin{array}{l}\text { A reduction in tumour size has } \\
\text { been associated with longer } \\
\text { PFS[195] } \\
14 \mathrm{mg} \text { QD with up-titration or } \\
18 \mathrm{mg} \text { QD without up-titration } \\
\text { potentially provides } \\
\text { comparable efficacy and } \\
\text { improved safety[196] }\end{array}$ \\
\hline Lorlatinib & Exploratory & NA & NA & $\begin{array}{l}\text { NA } \\
<70-125>[200]\end{array}$ & $\mathrm{No}[200,201]$ & $\begin{array}{l}\text { Yes } \\
\text { (hypercholesterol- }\end{array}$ & Yes[201] & $\begin{array}{l}\text { Different minimum effective } \\
\text { concentrations have been }\end{array}$ \\
\hline \multirow[t]{2}{*}{ Substance } & $\begin{array}{l}\text { Evidence } \\
\text { level for } \\
\text { TDM }\end{array}$ & $\begin{array}{l}\text { Proposed } \\
\text { TDM } \\
\text { target } \\
{[\mathrm{ng} / \mathrm{mL}]}\end{array}$ & $\begin{array}{l}\text { Patients } \\
\text { below TDM } \\
\text { target at } \\
\text { standard } \\
\text { dose }(\%) \\
\end{array}$ & $\begin{array}{l}\text { Reported } \\
\text { mean/median } \\
\text { CSS,min at }_{\text {standard dose }} \\
{[\mathrm{ng} / \mathrm{mL}]\left(\mathrm{IIV}^{\mathrm{a}}\right)} \\
\end{array}$ & $\begin{array}{l}\text { Exposure-response } \\
\text { relationship } \\
\text { [yes/no] } \\
\text { (associated } \\
\text { parameter(s)) } \\
\end{array}$ & $\begin{array}{l}\text { Exposure-safety } \\
\text { relationship } \\
\text { [yes/no] } \\
\text { (associated } \\
\text { parameter(s)) }\end{array}$ & $\begin{array}{l}\text { NLME } \\
\text { model } \\
\text { available }\end{array}$ & Comments \\
\hline & & & & & & $\begin{array}{l}\text { aemia, any } \mathrm{G} 3 / 4 \\
\mathrm{AEs})[200]\end{array}$ & & $\begin{array}{l}\text { reported for different ALK } \\
\text { mutations[202] }\end{array}$ \\
\hline Midostaurin & Exploratory & NA & NA & $\begin{array}{l}467(30 \%)[203] \\
(50 \mathrm{mg} \text { BID) }\end{array}$ & $\begin{array}{l}\text { Yes (blood blast } \\
\text { response)[206] }\end{array}$ & $\mathrm{No}[204]$ & Yes[207] & $\begin{array}{l}\text { A trend for higher probability } \\
\text { of response with higher } C_{S S, m i n} \\
\text { has been reported[204] }\end{array}$ \\
\hline & & & & $\begin{array}{l}919-1060[204] \\
(54 \%)[205] \\
(100 \mathrm{mg} \text { BID })\end{array}$ & & & & $\begin{array}{l}\text { A significantly increased risk } \\
\text { of death with lower exposure } \\
\text { to the metabolite CGP2221 has } \\
\text { been reported[204] }\end{array}$ \\
\hline Neratinib & Exploratory & NA & NA & $\begin{array}{l}\text { AUC: } 1,402^{b} \\
(56 \%)[208]\end{array}$ & $\begin{array}{l}\text { Yes (OR (only } \\
\text { monotherapy)) } \\
{[209]}\end{array}$ & No[209] & Yes[209] & \\
\hline Nintedanib & Exploratory & NA & NA & $\begin{array}{l}13.1^{\mathrm{e}} \\
(70 \%)[210]\end{array}$ & $\begin{array}{l}\text { Yes (sVEGFR } \\
\text { decrease)[211] }\end{array}$ & $\begin{array}{l}\text { Yes } \\
\text { (gastrointestinal } \\
\text { disorders)[210] }\end{array}$ & Yes[212] & $\begin{array}{l}\text { Weak exposure-response and } \\
\text { exposure-safety relationships } \\
\text { observed[213] }\end{array}$ \\
\hline Niraparib & Exploratory & NA & NA & $649 \pm 135^{\mathrm{f}}[214]$ & Yes (PFS)[215] & $\begin{array}{l}\text { Yes (thrombo- } \\
\text { cytopenia) }[216]\end{array}$ & Yes[216] & \\
\hline Olaparib & Exploratory & NA & NA & $\begin{array}{l}\text { Tablets: } 1570 \\
(86 \%)[217] \\
\text { Capsules: } 1860 \\
(84 \%)[217]\end{array}$ & Yes (PFS)[218] & $\begin{array}{l}\text { Yes (diarrhoea, } \\
\text { anaemia, decreased } \\
\text { appetite, dysgeusia, } \\
\text { fatigue, nausea, } \\
\text { vomiting) } \\
{[218,219]}\end{array}$ & Yes[217] & \\
\hline Osimertinib & Exploratory & NA & NA & $\begin{array}{l}166^{g}(49 \%) \\
{[220]}\end{array}$ & No[220,221] & $\begin{array}{l}\text { Yes (rash, } \\
\text { diarrhoea)[221] }\end{array}$ & Yes[222] & \\
\hline
\end{tabular}




\begin{tabular}{|c|c|c|c|c|c|c|c|c|}
\hline Substance & $\begin{array}{l}\text { Evidence } \\
\text { level for } \\
\text { TDM }\end{array}$ & $\begin{array}{l}\text { Proposed } \\
\text { TDM } \\
\text { target } \\
{[\mathrm{ng} / \mathrm{mL}]}\end{array}$ & $\begin{array}{l}\text { Patients } \\
\text { below TDM } \\
\text { target at } \\
\text { standard } \\
\text { dose }(\%) \\
\end{array}$ & $\begin{array}{l}\text { Reported } \\
\text { mean/median } \\
\text { Css,min at } \\
\text { standard dose } \\
{[\mathrm{ng} / \mathrm{mL}]\left(\mathrm{IIV}^{\mathrm{a}}\right)} \\
\end{array}$ & $\begin{array}{l}\text { Exposure-response } \\
\text { relationship } \\
\text { [yes/no] } \\
\text { (associated } \\
\text { parameter(s)) } \\
\end{array}$ & $\begin{array}{l}\text { Exposure-safety } \\
\text { relationship } \\
\text { [yes/no] } \\
\text { (associated } \\
\text { parameter(s)) } \\
\end{array}$ & $\begin{array}{l}\text { NLME } \\
\text { model } \\
\text { available }\end{array}$ & Comments \\
\hline Palbociclib & Exploratory & NA & NA & $61(42 \%)[223]$ & $\begin{array}{l}\text { Inconclusive (trend } \\
\text { for PFS)[223] }\end{array}$ & $\begin{array}{l}\text { Yes } \\
\text { (neutropenia) }[223,2 \\
24]\end{array}$ & Yes[225] & \\
\hline Panobinostat & Exploratory & NA & NA & $\begin{array}{l}\mathrm{AUC}_{0-24: 174^{\mathrm{b}}} \\
(92 \%)[226]\end{array}$ & $\begin{array}{l}\text { Yes (trend for } \\
\text { ORR)[227] }\end{array}$ & $\begin{array}{l}\text { Yes (thrombo- } \\
\text { cytopenia, } \\
\text { diarrhoea) [227] }\end{array}$ & Yes[228] & \\
\hline Pexidartinib & Exploratory & NA & NA & $\begin{array}{l}\mathrm{AUC}_{0-12}: 77465^{\mathrm{b}} \\
(32 \%)[229]\end{array}$ & $\begin{array}{l}\text { Inconclusive (trend } \\
\text { for ORR)[229] }\end{array}$ & $\begin{array}{l}\text { Yes (AST and ALT } \\
\text { elevation)[229] }\end{array}$ & Yes[229] & \\
\hline Ponatinib & Exploratory & NA & NA & $34(45 \%)[230]$ & $\begin{array}{l}\text { Inconclusive (trend } \\
\text { for McyR, MaHR) } \\
\text { [231] }\end{array}$ & $\begin{array}{l}\text { Yes (hypertension, } \\
\text { ischemia)[231] }\end{array}$ & No & $\begin{array}{l}\text { A dose intensity-safety } \\
\text { relationship was } \\
\text { identified[231] }\end{array}$ \\
\hline Regorafenib & Exploratory & NA & NA & $\begin{array}{l}1400(57 \%) \\
{[232]}\end{array}$ & $\begin{array}{l}\text { Yes (sVEGFR2 } \\
\text { level decrease)[232] }\end{array}$ & $\begin{array}{l}\text { Yes (rash, } \\
\text { bilirubin) }[233,234]\end{array}$ & Yes[235] & \\
\hline Ribociclib & Exploratory & NA & NA & $732(91 \%)$ [236] & Inconclusive[236] & $\begin{array}{l}\text { Yes }(\mathrm{QTcF} \\
\text { prolongation, } \\
\text { neutropenia)[236] }\end{array}$ & Yes[236] & $\begin{array}{l}\text { Due to lack of data, an } \\
\text { exposure-response analysis } \\
\text { could not be performed[236] }\end{array}$ \\
\hline Rucaparib & Exploratory & NA & NA & $\begin{array}{l}1754 \pm 805 \\
{[237]}\end{array}$ & $\begin{array}{l}\text { Inconclusive } \\
\text { (RECIST response } \\
\text { rate) }[238]\end{array}$ & $\begin{array}{l}\text { Yes (creatinine, } \\
\text { ALT and AST } \\
\text { elevation, fatigue, } \\
\text { thrombo- } \\
\text { cytopenia)[237] }\end{array}$ & Yes[237] & \\
\hline Ruxolitinib & Exploratory & NA & NA & $\begin{array}{l}5.52 \pm 5.82^{\mathrm{h}} \\
(10 \mathrm{mg} \mathrm{BID}) \\
11.4 \pm 6.01^{\mathrm{h}} \\
(15 \mathrm{mg} \mathrm{BID}) \\
{[239]}\end{array}$ & $\begin{array}{l}\text { Yes (spleen volume } \\
\text { reduction, total } \\
\text { symptom score) } \\
{[239]}\end{array}$ & $\begin{array}{l}\text { Yes (reduced } \\
\text { platelet count, } \\
\text { reduced } \\
\text { haemoglobin)[239] }\end{array}$ & Yes[240] & \\
\hline Sorafenib & Exploratory & NA & NA & $\begin{array}{l}3750(104 \%) \\
{[241]}\end{array}$ & $\begin{array}{l}\text { Yes (PFS, } \\
\text { OS)[242,243] }\end{array}$ & $\begin{array}{l}\text { Yes (G3/4 AEs, } \\
\text { HFS, }\end{array}$ & Yes[247] & $\begin{array}{l}\text { Exposure has been reported to } \\
\text { decrease over time [244] }\end{array}$ \\
\hline \multirow[t]{2}{*}{ Substance } & $\begin{array}{l}\text { Evidence } \\
\text { level for } \\
\text { TDM }\end{array}$ & $\begin{array}{l}\text { Proposed } \\
\text { TDM } \\
\text { target } \\
{[\mathrm{ng} / \mathrm{mL}]}\end{array}$ & $\begin{array}{l}\text { Patients } \\
\text { below TDM } \\
\text { target at } \\
\text { standard } \\
\text { dose }(\%) \\
\end{array}$ & $\begin{array}{l}\text { Reported } \\
\text { mean/median } \\
\text { Css,min at } \\
\text { standard dose } \\
{[\mathrm{ng} / \mathrm{mL}]\left(\mathrm{IIV}^{\mathrm{a}}\right)} \\
\end{array}$ & $\begin{array}{l}\text { Exposure-response } \\
\text { relationship } \\
\text { [yes/no] } \\
\text { (associated } \\
\text { parameter(s)) } \\
\end{array}$ & $\begin{array}{l}\text { Exposure-safety } \\
\text { relationship } \\
\text { [yes/no] } \\
\text { (associated } \\
\text { parameter(s)) } \\
\end{array}$ & $\begin{array}{l}\text { NLME } \\
\text { model } \\
\text { available }\end{array}$ & Comments \\
\hline & & & & & & $\begin{array}{l}\text { hypertension) }[242- \\
246]\end{array}$ & & \\
\hline Talazoparib & Exploratory & NA & NA & $\begin{array}{l}3.54(63 \%) \\
{[248]}\end{array}$ & Yes (PFS) $[248,249]$ & $\begin{array}{l}\text { Yes (G3 AEs, } \\
\text { anaemia, } \\
\text { thrombocytopenia) } \\
{[248,249]}\end{array}$ & Yes[250] & \\
\hline Tivozanib & Exploratory & NA & NA & $\begin{array}{l}\text { NA } \\
<16.0- \\
30.9>[251]\end{array}$ & $\begin{array}{l}\text { Yes (tumour } \\
\text { shrinkage, } \\
\text { PFS)[251] }\end{array}$ & $\begin{array}{l}\text { Yes (hypertension, } \\
\text { HFS) [251] }\end{array}$ & Yes[251] & $\begin{array}{l}\text { - The exposure-response } \\
\text { relationship described in [251] } \\
\text { is convincing: PFS was } 26.4 \\
\text { weeks vs } 72.1 \text { weeks in } \\
\text { patients with } \mathrm{C}_{\text {avg }} \text { in the lowest } \\
\text { vs highest quartile, } \\
\text { respectively }\end{array}$ \\
\hline Vandetanib & Exploratory & NA & NA & 795 (NA) [252] & $\begin{array}{l}\text { Yes (best calcitonin } \\
\text { response) }[252]\end{array}$ & $\begin{array}{l}\text { Yes (diarrhoea, } \\
\text { fatigue, QTc } \\
\text { prolongation) [252] }\end{array}$ & Yes[252] & $\begin{array}{l}\text { - } \mathrm{IC}_{50} \text { values }(190 \mathrm{ng} / \mathrm{mL}) \text { have } \\
\text { been used to support dose } \\
\text { selection in early clinical } \\
\text { trials[253] }\end{array}$ \\
\hline Bicalutamide & $\begin{array}{l}\text { Not } \\
\text { recommended }\end{array}$ & NA & NA & $\begin{array}{l}8850<1380- \\
21700>(50 \mathrm{mg} \\
\text { QD) }[254]\end{array}$ & $\mathrm{No}[256]$ & $\mathrm{No}[256]$ & No & \\
\hline & & & & $\begin{array}{l}16400<2400- \\
44600>(150 \mathrm{mg} \\
\text { QD) }[255]\end{array}$ & & & & \\
\hline Cobimetinib & $\begin{array}{l}\text { Not } \\
\text { recommended }\end{array}$ & NA & NA & $127(87 \%)[257]$ & $\mathrm{No}[257]$ & No[257] & Yes[258] & \\
\hline Darolutamide & $\begin{array}{l}\text { Not } \\
\text { recommended }\end{array}$ & NA & NA & 3780 (NA)[259] & NA & NA & Yes[259] & \\
\hline
\end{tabular}




\begin{tabular}{|c|c|c|c|c|c|c|c|c|}
\hline Substance & $\begin{array}{l}\text { Evidence } \\
\text { level for } \\
\text { TDM }\end{array}$ & $\begin{array}{l}\text { Proposed } \\
\text { TDM } \\
\text { target } \\
{[\mathrm{ng} / \mathrm{mL}]}\end{array}$ & $\begin{array}{l}\text { Patients } \\
\text { below TDM } \\
\text { target at } \\
\text { standard } \\
\text { dose }(\%) \\
\end{array}$ & $\begin{array}{l}\text { Reported } \\
\text { mean/median } \\
\text { Css,min at } \\
\text { standard dose } \\
{[\mathrm{ng} / \mathrm{mL}]\left(\mathrm{IIV}^{\mathrm{a}}\right)} \\
\end{array}$ & $\begin{array}{l}\text { Exposure-response } \\
\text { relationship } \\
\text { [yes/no] } \\
\text { (associated } \\
\text { parameter(s)) } \\
\end{array}$ & $\begin{array}{l}\text { Exposure-safety } \\
\text { relationship } \\
\text { [yes/no] } \\
\text { (associated } \\
\text { parameter(s)) }\end{array}$ & $\begin{array}{l}\text { NLME } \\
\text { model } \\
\text { available }\end{array}$ & Comments \\
\hline Duvelisib & $\begin{array}{l}\text { Not } \\
\text { recommended }\end{array}$ & NA & NA & $\begin{array}{l}\mathrm{AUC}_{0-12}: 7888^{\mathrm{b}} \\
(77 \%)[260]\end{array}$ & $\mathrm{No}[261]$ & $\mathrm{No}[261]$ & No & \\
\hline Entrectinib & $\begin{array}{l}\text { Not } \\
\text { recommended }\end{array}$ & NA & NA & $\begin{array}{l}\text { NA } \\
<812-872>i \\
{[262]}\end{array}$ & No & $\begin{array}{l}\text { Inconclusive (trend } \\
\text { for probability of } \\
\text { AE Grade } \geq 3 \text { ) [262] }\end{array}$ & Yes[262] & \\
\hline Enzalutamide & $\begin{array}{l}\text { Not } \\
\text { recommended }\end{array}$ & NA & NA & $\begin{array}{l}11400(26 \%) \\
{[263]}\end{array}$ & $\mathrm{No}[263,264]$ & $\mathrm{No}[263]$ & Yes[263] & \\
\hline Exemestane & $\begin{array}{l}\text { Not } \\
\text { recommended }\end{array}$ & NA & NA & $\begin{array}{l}4.1[1.3-38.1]^{\mathrm{j}} \\
{[14]}\end{array}$ & NA & NA & Yes[265] & \\
\hline Idelalisib & $\begin{array}{l}\text { Not } \\
\text { recommended }\end{array}$ & NA & NA & 426 (NA) [266] & $\mathrm{No}[266-268]$ & $\mathrm{No}[266-268]$ & Yes[269] & \\
\hline Lapatinib & Exploratory & NA & NA & $\begin{array}{l}490\{50-5230\} \\
(1000 \mathrm{mg} \\
\text { QD) }[270] \\
635^{\mathrm{k}}(\mathrm{NA}) \\
(1250 \mathrm{mg}) \\
780\{520-1180\} \\
(1500 \mathrm{mg} \\
\text { QD) }[271]\end{array}$ & NA & NA & Yes[272] & $\begin{array}{l}\text { No exposure-response or } \\
\text { exposure-safety relationships } \\
\text { established but majority of } \\
\text { responders reported to have } \\
\mathrm{C}_{\mathrm{SS}, \mathrm{min}} 300-600 \mathrm{ng} / \mathrm{mL}[270]\end{array}$ \\
\hline Larotrectinib & $\begin{array}{l}\text { Not } \\
\text { recommended }\end{array}$ & NA & NA & $5.14^{1}(\mathrm{NA})[273]$ & $\mathrm{No}[274]$ & $\mathrm{No}[274]$ & Yes[274] & \\
\hline Venetoclax & $\begin{array}{l}\text { Not } \\
\text { recommended }\end{array}$ & NA & NA & 520 (NA)[275] & $\begin{array}{l}\text { Inconclusive (trend } \\
\text { for CR)[276] }\end{array}$ & No[276] & Yes[277] & \\
\hline Substance & $\begin{array}{l}\text { Evidence } \\
\text { level for } \\
\text { TDM }\end{array}$ & $\begin{array}{l}\text { Proposed } \\
\text { TDM } \\
\text { target } \\
{[\mathrm{ng} / \mathrm{mL}]}\end{array}$ & $\begin{array}{l}\text { Patients } \\
\text { below TDM } \\
\text { target at } \\
\text { standard } \\
\text { dose (\%) } \\
\end{array}$ & $\begin{array}{l}\text { Reported } \\
\text { mean/median } \\
\text { Css,min at } \\
\text { standard dose } \\
{[\mathrm{ng} / \mathrm{mL}]\left(\mathrm{IIV}^{\mathrm{a}}\right)} \\
\end{array}$ & $\begin{array}{l}\text { Exposure-response } \\
\text { relationship } \\
\text { [yes/no] } \\
\text { (associated } \\
\text { parameter(s)) } \\
\end{array}$ & $\begin{array}{l}\text { Exposure-safety } \\
\text { relationship } \\
\text { [yes/no] } \\
\text { (associated } \\
\text { parameter(s)) }\end{array}$ & $\begin{array}{l}\text { NLME } \\
\text { model } \\
\text { available }\end{array}$ & Comments \\
\hline Vismodegib & $\begin{array}{l}\text { Not } \\
\text { recommended }\end{array}$ & NA & NA & $\begin{array}{l}11367 \pm 4084^{\mathrm{m}} \\
{[278]}\end{array}$ & $\mathrm{No}[278]$ & $\mathrm{No}[278]$ & Yes[278] & \\
\hline Vorinostat & $\begin{array}{l}\text { Not } \\
\text { recommended }\end{array}$ & NA & NA & $\begin{array}{l}\mathrm{AUC}_{0-\infty}: 1430^{\mathrm{b}, \mathrm{n}} \\
\text { (NA)[279] }\end{array}$ & $\begin{array}{l}\text { Inconclusive (trend } \\
\text { for ORR) [280] }\end{array}$ & $\begin{array}{l}\text { Inconclusive (trend } \\
\text { for QTc } \\
\text { prolongation) }[280]\end{array}$ & No & \\
\hline
\end{tabular}

${ }^{\mathrm{Na}}$ Interindividual variability in mean/median exposure is reported as (CV\%), [interquartile range], \pm standard deviation, $\{95 \% \mathrm{CI}\}$ or $<$ range $>$, according to the available data.

${ }^{\mathrm{b}} \mathrm{AUC}$ units are $\mathrm{ng} * \mathrm{~h} / \mathrm{mL}$.

'Target concentrations are the sum of concentrations of both sunitinib and its active metabolite N-desethylsunitnib.

${ }^{\mathrm{d}}$ Target concentration and mean/median exposure for tamoxifen are reported for tamoxifen's most active metabolite endoxifen.

${ }^{e}$ Calculated based on the dose-normalised steady-state trough concentration of $0.065 \mathrm{ng} / \mathrm{ml} / \mathrm{mg}$ and a dose of $200 \mathrm{mg}$ BID[210].

${ }^{\mathrm{f}}$ Calculated based on the the weighted average of two groups $(\mathrm{A}: \mathrm{n}=10, \mathrm{~B}: \mathrm{n}=26)$ at the standard dose $[\mathrm{nmol} / \mathrm{L}]$ and the molecular weight of $320.4 \mathrm{~g} / \mathrm{mol}$.

${ }^{g}$ Calculated based on the reported concentrations in $\mathrm{nM}$ and the molecular weight of $499.6 \mathrm{~g} / \mathrm{mol}$.

${ }^{\mathrm{h}}$ Calculated based on the reported concentrations in $\mathrm{nM}$ and the molecular weight of $306.4 \mathrm{~g} / \mathrm{mol}$.

${ }^{\mathrm{i}}$ Calculated from the predicted PK exposures [nM] in two simulation scenarios (1: weight effect on clearance, 2: allometric scaling) using a molecular weight of 560.6 g/mol.

${ }^{\mathrm{j}}$ Calculated based on median $\mathrm{C}_{\max }\left(7.7 \mathrm{ng} / \mathrm{mL}\right.$, range 2.5-72,0)[281] and $\mathrm{t}_{1 / 2}$, calculated with the formula proposed by Wang et al. [10].

${ }^{\mathrm{k}}$ Calculated based on the reported $\mathrm{C}_{\min }$ at $1500 \mathrm{mg}$ and $1000 \mathrm{mg}$ and dose-linear pharmacokinetics[282].

${ }^{1}$ Calculated based on the reported unbound concentration in $\mathrm{nM}$ and the molecular weight of $428.4 \mathrm{~g} / \mathrm{mol}$.

${ }^{\mathrm{m}}$ Calculated based on the reported concentrations in $\mathrm{nM}$ and the molecular weight of $421.3 \mathrm{~g} / \mathrm{mol}$.

${ }^{\mathrm{n}}$ Calculated based on the reported concentrations in $\mathrm{nM}$ and the molecular weight of $264.3 \mathrm{~g} / \mathrm{mol}$.

Abbreviations: $A E$ : adverse event; $A L B$ : albumin; $A L T$ : alanine aminotransferase; $A S T$ : aspartate aminotransferase; $A U C$ : area under the concentration-time curve; $B I D$ : twice daily; $B O R$ : best objective response; $B T K$ : Bruton's tyrosine kinase; $C C y R$ : complete cytogenetic response; $C I$ : confidence interval; $C K$ : creatinine kinase; $C_{a v g . s s}$ average concentration at steady-state; $C_{S S \text { min }}$ : minimum concentration at steady-state; $C_{S S}$ : concentration in steady state, no further specification of sampling time; $C H R$ : complete hematologic response; $C M L$ : chronic myeloid leukemia; $C R$ : complete response; $d B P$ : diastolic blood pressure; Gx: grade x; GIST: gastrointestinal stromal tumours, HFS: handfoot syndrome; $I I V$ : interindividual variability; $I C_{50}$ : half maximal inhibitory concentration; $I R R$ : independent radiology review; $M a H R$ : major hematologic response; $M C y R$ : major cytogenetic response; $M M R$ : major molecular response; $M R$ : molecular remission; $N A$ : not available; OR: objective response; ORR: objective response rate; $O S$ : overall survival; PFS: progression-free survival; $P K$ : pharmacokinetics; $P N$ : peripheral neuropathy; $P R$ : partial response; $Q D$ : once daily; RCC: renal cell carcinoma; RECIST: Response evaluation criteria in solid tumors; RR: recurrence rate; SoC: standard of care; STAT3: Signal transducer and activator of transcription 3; sVEGFR: soluble vascular endothelial growth factor receptor; TDM: therapeutic drug monitoring; TTP: time to progression

pazopanib dosing, $57 \%$ of all patients $(n=30)$ showed pazopanib $\mathrm{C}_{\mathrm{SS} \text {, } \min }<20 \mathrm{mg} / \mathrm{L}$ under standard treatment and $41 \%$ of these successfully achieved therapeutic $\mathrm{C}_{\mathrm{SS} \text {, min }}$ upon dose increases to 1000-1800 mg QD [48]. Furthermore, all patients who achieved a partial response showed $\mathrm{C}_{\mathrm{SS}, \min } \geq$
$20 \mathrm{mg} / \mathrm{mL}$. In a recent retrospective observational clinical study in 27 RCC patients, a significant correlation between pazopanib $\mathrm{C}_{\mathrm{SS}, \min } \geq 20.5 \mathrm{mg} / \mathrm{mL}$ and objective response was established [77]. Based on the evidence for an exposureresponse relationship and the proven feasibility of 
individualised dosing, we recommend TDM-guided pazopanib dosing, targeting plasma $\mathrm{C}_{\mathrm{SS}, \min } \geq 20 \mathrm{mg} / \mathrm{mL}$. A published population PK model [78] can be used in a MIPD framework for pazopanib. However, due to a dose-dependent decrease in the relative BA of pazopanib, conventional dose increases are an inefficient strategy to increase exposure. Alternative cost-neutral strategies have been described in literature. Splitting intake moments (i.e. $400 \mathrm{mg}$ BID instead of $800 \mathrm{mg}$ QD) resulted in a $79 \%$ increase in $\mathrm{C}_{\min }$ [43]. Moreover, concomitant intake with food successfully increased exposure as well [42].

\section{Sunitinib}

Significant increases in toxicities in patients with sunitinib + active metabolite SU012662 $\mathrm{C}_{\mathrm{SS}, \mathrm{min}} \geq 100 \mathrm{ng} / \mathrm{mL}$ have been reported [79, 80]. For RCC patients, an efficacy PK target of $50-100 \mathrm{ng} / \mathrm{mL}$ has been proposed in intermittent dosing at $50 \mathrm{mg}$ QD [80]. Exploiting dose linearity, this target was extrapolated to $\mathrm{C}_{\mathrm{SS}, \min } \geq 37.5 \mathrm{ng} / \mathrm{mL}$ for continuous dosing at $37.5 \mathrm{mg}$ QD in GIST patients [12]. Based on a summary of exposure-response analyses [60], TDM-guided sunitinib dosing targeting a sunitinib + SU012662 $\mathrm{C}_{\mathrm{SS} \text {, min }}$ of 50 $100 \mathrm{ng} / \mathrm{mL}$ was prospectively tested in a clinical study in 43 patients with advanced solid malignancies [47]. Of the patients eligible for PK-evaluation $(n=29), 52 \%(n=14)$ showed sunitinib $+\mathrm{SU} 012662 \mathrm{C}_{\mathrm{SS}, \min }<50 \mathrm{ng} / \mathrm{mL}$ at treatment initiation, and among those, 5 patients reached therapeutic total trough levels after dose escalation without experiencing additional toxicities. These findings underline both the need and feasibility of TDM-guided sunitinib dosing, for which a published population PK/PD model can be used [81]. Biomarkers such as the soluble vascular endothelial growth factor receptor may provide additional information on individual response and have been integrated into PK/PD models [82]. Because of the different half-lives of sunitinib and SU012662, $\mathrm{C}_{\mathrm{SS} \text {,min }}$ should be calculated separately when using the log-linear extrapolation method. Due to the long half-lives and time to reach steady-state, it is important to collect PK samples in the last treatment week before the offtreatment period.

\section{Tamoxifen}

Compared to higher values, $\mathrm{C}_{\mathrm{SS}}$ of $<5.97 \mathrm{ng} / \mathrm{mL}[83]$ and $<$ $5.2 \mathrm{ng} / \mathrm{mL}$ [84] of tamoxifen's active metabolite endoxifen have been associated with more additional breast cancer events and shorter distant relapse-free survival, respectively. While body weight and age have a significant impact as well [85], CYP2D6 phenotype accounts for $18-43 \%$ of the observed IIV of $40-49 \%$ in endoxifen $C_{S S}$ [14]. Considering this, TDM of endoxifen might be promising to identify patients with sub-optimal target concentrations [86]. Because no toxic tamoxifen dose has been identified, dose increases up until $120 \mathrm{mg}$ QD for patients with endoxifen $\mathrm{C}_{\mathrm{SS}}<5.97 \mathrm{ng} /$ $\mathrm{mL}$ have been investigated and TDM has proven feasible [20, 87-92]. As it takes about 3 months to attain endoxifen steady state, we propose to use MIPD for early endoxifen target attainment [92].

\section{Discussion}

While exposure-response and exposure-safety relationships have been observed for many OADs, viable PK targets are only available for a few. Future clinical and 'real-world' studies are needed to identify clear target ranges associated with favourable outcome. More PK/PD analyses conducted in (pre-)clinical development could help to characterise exposure-response relationships earlier. More focus must also be dedicated on the establishment of TDM as part of routine patient care. This might be challenging, as bio-analytical assays should be available and a solid logistic system with a short turnaround time in place. At the Netherlands Cancer Institute, TDM has been implemented in routine care, and PK samples are collected at routine visits to the outpatient clinic. Concentrations of 35 different OADs are measured weekly using liquid chromatography-tandem mass spectrometry [93-96], treatment recommendations are reported within $24 \mathrm{~h}$ to the treating physician and results can be discussed with patients $1-2$ weeks after their visit. This approach is also emerging in Australia with several OADs being measured on request with current efficacy-implementation studies underway. Additional data should be prospectively collected (i.e. in registries) to further investigate the effect of TDM on treatment outcomes. Novel microsampling techniques, i.e. volumetric absorptive microsampling (VAMS) [97], could help to provide the TDM results even before patients visit the outpatient clinic. VAMS allows to precisely sample a small volume of capillary blood from the fingertip with a dedicated sampling device. After blood collection, the device is dried in the open air and shipped to a laboratory via regular mail without pre-processing or cooling during transport. Given its easy and minimally invasive character, this technique shows high potential: in the future, VAMS samples could be obtained at home and shipped to a laboratory by patients themselves. Upon sample analysis, results would be communicated to the treating oncologist and discussed with the patient at the next visit. Of note, the disadvantages of VAMS are not fully elucidated yet. Current limitations are variable analyte recoveries dependent on haematocrit [98] and the time-consuming determination of capillary-tovenous blood conversion factors, needed to compare measured capillary whole blood with venous plasma target concentrations [97]. Furthermore, to make this approach feasible, a wellconnected infrastructure of oncologists, laboratories and PKspecialists must be available. 


\section{Conclusion}

In this review, we summarised the opportunities and challenges associated with TDM of OADs and outlined different TDM approaches, their respective advantages and disadvantages. We provided strong arguments why routine TDM should be established as a part of OAD treatment and reviewed the available evidence for all oral targeted antineoplastic drugs currently approved by the EMA and/or FDA. Finally, we provided an outlook into the future and proposed a strategy to increase feasibility and acceptance of TDM as part of routine clinical care.

Code availability Not applicable.

Authors' contributions AM, OS, UJ and CK developed the research question. AM, OS and SLG conducted the searches and extracted the data. AM, OS and SLG analysed the data and did the quality assessment. AM wrote the first draft of the manuscript. All authors discussed the analysis results, contributed to the revision of the manuscript and reviewed and approved the final version.

Funding Open Access funding enabled and organized by Projekt DEAL.

Data availability Not applicable.

\section{Compliance with ethical standards}

Conflict of interest $\mathrm{CK}$ and $\mathrm{WH}$ report grants from an industry consortium (AbbVie Deutschland GmbH \& Co. KG, Astra Zeneca, Boehringer Ingelheim Pharma GmbH \& Co. KG, Grünenthal GmbH, F. Hoffmann-La Roche Ltd., Merck KGaA and Sanofi) for the PharMetrX PhD program. CK reports grant for the Innovative Medicines Initiative-Joint Undertaking ('DDMoRe'). CK and RM report grants from the Federal Ministry of Education and Research within the Joint Programming Initiative on Antimicrobial Resistance Initiative (JPIAMR), all outside the submitted work. OS reports endowed professorship grant (Horphag research Ltd) and funding for the project 'Individualized cancer therapy with kinase inhibitors using drug monitoring - optimization by minimally invasive at-home sampling' (Hector Stiftung II gGmbH). The remaining authors declare that the research was conducted in absence of any commercial or financial relationships that could be construed as a potential conflict of interest.

Ethics approval Not applicable.

Consent to participate Not applicable.

Consent for publication Not applicable.

Open Access This article is licensed under a Creative Commons Attribution 4.0 International License, which permits use, sharing, adaptation, distribution and reproduction in any medium or format, as long as you give appropriate credit to the original author(s) and the source, provide a link to the Creative Commons licence, and indicate if changes were made. The images or other third party material in this article are included in the article's Creative Commons licence, unless indicated otherwise in a credit line to the material. If material is not included in the article's Creative Commons licence and your intended use is not permitted by statutory regulation or exceeds the permitted use, you will need to obtain permission directly from the copyright holder. To view a copy of this licence, visit http://creativecommons.org/licenses/by/4.0/.

\section{References}

1. Committee for Medicinal Products for Human Use (CHMP) European Medicines Agency (2019) Glivec European public assessment report. https://www.ema.europa.eu/documents/productinformation/glivec-epar-product-information_en.pdf.

2. van Erp NP, Gelderblom H, Guchelaar HJ (2009) Clinical pharmacokinetics of tyrosine kinase inhibitors. Cancer Treat Rev 35: 692-706. https://doi.org/10.1016/j.ctrv.2009.08.004

3. Klümpen HJ, Samer CF, Mathijssen RHJ, Schellens JHM, Gurney H (2011) Moving towards dose individualization of tyrosine kinase inhibitors. Cancer Treat Rev 37:251-260. https://doi. org/10.1016/j.ctrv.2010.08.006

4. Zhao YY, Li S, Zhang Y, Zhao HY, Liao H, Guo Y, Shi YX, Jiang W, Xue C, Zhang L (2011) The relationship between drug exposure and clinical outcomes of non-small cell lung cancer patients treated with gefitinib. Med Oncol 28:697-702. https://doi.org/10. 1007/s12032-010-9541-0

5. Le Tourneau C, Lee JJ, Siu LL (2009) Dose escalation methods in phase I cancer clinical trials. J Natl Cancer Inst 101:708-720. https://doi.org/10.1093/jnci/djp079

6. van Brummelen EMJ, Huitema ADR, van Werkhoven E, Beijnen JH, Schellens JHM (2016) The performance of model-based versus rule-based phase I clinical trials in oncology: a quantitative comparison of the performance of model-based versus rule-based phase I trials with molecularly targeted anticancer drugs over the last 2 years. J Pharmacokinet Pharmacodyn 43:235-242. https:// doi.org/10.1007/s10928-016-9466-0

7. Groenland SL, Mathijssen RHJ, Beijnen JH, Huitema ADR, Steeghs N (2019) Individualized dosing of oral targeted therapies in oncology is crucial in the era of precision medicine. Eur J Clin Pharmacol. https://doi.org/10.1007/s00228-019-02704-2

8. Cardoso E, Csajka C, Schneider MP, Widmer N (2018) Effect of adherence on pharmacokinetic/pharmacodynamic relationships of oral targeted anticancer drugs. Clin Pharmacokinet 57:1-6. https:// doi.org/10.1007/s40262-017-0571-z

9. Partridge AH (2002) Adherence to therapy with oral antineoplastic agents. CancerSpectrum Knowl Environ 94:652-661. https:// doi.org/10.1093/jnci/94.9.652

10. Wang Y, Chia YL, Nedelman J, Schran H, Mahon FX, Molimard $\mathrm{M}$ (2009) A therapeutic drug monitoring algorithm for refining the imatinib trough level obtained at different sampling times. Ther Drug Monit 31:579-584. https://doi.org/10.1097/ftd. 0b013e3181b2c8cf

11. Gao B, Yeap S, Clements A, Balakrishnar B, Wong M, Gurney H (2012) Evidence for therapeutic drug monitoring of targeted anticancer therapies. J Clin Oncol 30:4017-4025. https://doi.org/10. 1200/jco.2012.43.5362

12. Yu H, Steeghs N, Nijenhuis CM, Schellens JHM, Beijnen JH, Huitema ADR (2014) Practical guidelines for therapeutic drug monitoring of anticancer tyrosine kinase inhibitors: focus on the pharmacokinetic targets. Clin Pharmacokinet 53:305-325. https:// doi.org/10.1007/s40262-014-0137-2

13. Verheijen RB, Yu H, Schellens JHM, Beijnen JH, Steeghs N, Huitema ADR (2017) Practical recommendations for therapeutic drug monitoring of kinase inhibitors in oncology. Clin Pharmacol Ther 102:765-776. https://doi.org/10.1002/cpt.787

14. Groenland SL, van Nuland M, Verheijen RB, Schellens JHM, Beijnen JH, Huitema ADR, Steeghs N (2018) Therapeutic drug monitoring of oral anti-hormonal drugs in oncology. Clin Pharmacokinet https://doi.org/10.1007/s40262-018-0683-0

15. Sinnollareddy MG, Cotta MO, Roberts JA (2017) Pharmacokinetic and pharmacodynamic tools to increase efficacy. In: Pulcini C, Ergönül Ö, Can F, Beovic B (eds) Antimicrobial stewardship, 1st edn. Elsevier Inc, Amsterdam, pp 85-98 
16. Wicha SG, Kees MG, Solms A, Minichmayr IK, Kratzer A, Kloft C (2015) TDMx: a novel web-based open-access support tool for optimising antimicrobial dosing regimens in clinical routine. Int $\mathrm{J}$ Antimicrob Agents 45:442-444. https://doi.org/10.1016/j. ijantimicag.2014.12.010

17. Velghe S, Stove CP (2018) Volumetric absorptive microsampling as an alternative tool for therapeutic drug monitoring of firstgeneration anti-epileptic drugs (Analytical and Bioanalytical Chemistry, (2018), 410, 9, (2331-2341), 10.1007/s00216-0180866-4). Anal Bioanal Chem 410:2449. https://doi.org/10.1007/ s00216-018-0951-8

18. Gotta V, Widmer N, Decosterd LA, Chalandon Y, Heim D, Gregor M, Benz R, Leoncini-Franscini L, Baerlocher GM, Duchosal MA, Csajka C, Buclin T (2014) Clinical usefulness of therapeutic concentration monitoring for imatinib dosage individualization: results from a randomized controlled trial. Cancer Chemother Pharmacol 74:1307-1319. https://doi.org/10.1007/ s00280-014-2599-1

19. Cabel L, Blanchet B, Thomas-Schoemann A, Huillard O, Bellesoeur A, Cessot A, Giroux J, Boudou-Rouquette P, Coriat R, Vidal M, Saidu NEB, Golmard L, Alexandre J, Goldwasser F (2018) Drug monitoring of sunitinib in patients with advanced solid tumors: a monocentric observational French study. Fundam Clin Pharmacol 32:98-107. https://doi.org/10.1111/fcp. 12327

20. Fox P, Balleine RL, Lee C, Gao B, Balakrishnar B, Menzies AM, Yeap SH, Ali SS, Gebski V, Provan P, Coulter S, Liddle C, Hui R, Kefford R, Lynch J, Wong M, Wilcken N, Gurney H (2016) Dose escalation of tamoxifen in patients with low endoxifen level: evidence for therapeutic drug monitoring - the TADE study. Clin Cancer Res 22:3164-3171. https://doi.org/10.1158/1078-0432. ccr-15-1470

21. Deppenweiler M, Falkowski S, Saint-Marcoux F, Monchaud C, Picard N, Laroche ML, Tubiana-Mathieu N, Venat-Bouvet L, Marquet P, Woillard JB (2017) Towards therapeutic drug monitoring of everolimus in cancer? Results of an exploratory study of exposure-effect relationship. Pharmacol Res 121:138-144. https:// doi.org/10.1016/j.phrs.2017.04.029

22. Decosterd LA, Widmer N, Zaman K, Cardoso E, Buclin T, Csajka $C$ (2015) Therapeutic drug monitoring of targeted anticancer therapy. Biomark Med 9:887-893. https://doi.org/10.2217/bmm.15. 78

23. Chatelut E, Bruno R, Ratain MJ (2018) Intraindividual pharmacokinetic variability: focus on small-molecule kinase inhibitors. Clin Pharmacol Ther 103:956-958. https://doi.org/10.1002/cpt.937

24. Liefaard L, Chen C (2015) Quantifying the effectiveness of dose individualization by simulation for a drug with moderate pharmacokinetic variability. Ther Drug Monit 37

25. Alnaim L (2007) Therapeutic drug monitoring of cancer chemotherapy. J Oncol Pharm Pract 13:207-221. https://doi.org/10. 2165/00003088-199018040-00004

26. Rousseau A, Marquet P (2002) Application of pharmacokinetic modelling to the routine therapeutic drug monitoring of anticancer drugs. Fundam Clin Pharmacol 16:253-262. https://doi.org/10. 1046/j.1472-8206.2002.00086.x

27. Donagher J, Barras MA (2018) Therapeutic drug monitoring: using Bayesian methods to evaluate hospital practice. J Pharm Pract Res 48:522-529. https://doi.org/10.1002/jppr.1432

28. Donagher J, Martin JH, Barras MA (2017) Individualised medicine: why we need Bayesian dosing. Intern Med J 47:593-600. https://doi.org/10.1111/imj.13412

29. De Jonge ME, Huitema ADR, Schellens JHM, Rodenhuis S, Beijnen JH (2005) Individualised cancer chemotherapy: strategies and performance of prospective studies on therapeutic drug monitoring with dose adaptation: A review. Clin Pharmacokinet 44: 147-173. https://doi.org/10.2165/00003088-200544020-00002
30. Darwich AS, Ogungbenro K, Hatley OJ, Rostami-Hodjegan A (2017) Role of pharmacokinetic modeling and simulation in precision dosing of anticancer drugs. Transl Cancer Res 6:S1512S1529. https://doi.org/10.21037/tcr.2017.09.14

31. Aarons L, Ogungbenro K (2010) Optimal design of pharmacokinetic studies. Basic Clin Pharmacol Toxicol 106:250-255. https:// doi.org/10.1111/j.1742-7843.2009.00533.x

32. Drennan P, Doogue M, van Hal SJ, Chin P (2018) Bayesian therapeutic drug monitoring software: past, present and future. Int $\mathrm{J}$ Pharm 3:109-114. https://doi.org/10.4155/ipk-2018-0005

33. Holford NHG, Buclin T (2012) Safe and effective variability-a criterion for dose individualization. Ther Drug Monit 34:565-568. https://doi.org/10.1097/ftd.0b013e31826aabc3

34. Abrantes JA, Jönsson S, Karlsson MO, Nielsen EI (2019) Handling interoccasion variability in model-based dose individualization using therapeutic drug monitoring data. Br J Clin Pharmacol 85:1326-1336. https://doi.org/10.1111/bcp.13901

35. Keutzer L, Simonsson USH (2020) Individualized dosing with high inter-occasion variability is correctly handled with modelinformed precision dosing - using rifampicin as an example. Front Pharmacol 11:1-15. https://doi.org/10.3389/fphar.2020. 00794

36. Janssen JM, Dorlo TPC, Beijnen JH, Huitema ADR Evaluation of extrapolation methods to predict trough concentrations to guide therapeutic drug monitoring of oral anticancer drugs. Ther Drug Monit. https://doi.org/10.1097/ftd.0000000000000767

37. Herbrink M, Nuijen B, Schellens JHM, Beijnen JH (2017) Hightech drugs in creaky formulations. Pharm Res 34:1751-1753. https://doi.org/10.1007/s1 1095-017-2185-4

38. Josephs DH, Fisher DS, Spicer J, Flanagan RJ (2013) Clinical pharmacokinetics of tyrosine kinase inhibitors: implications for therapeutic drug monitoring. Ther Drug Monit 35:562-587. https://doi.org/10.1097/ftd.0b013e318292b931

39. Ribba B, Holford NH, Magni P, Trocóniz I, Gueorguieva I, Girard P, Sarr C, Elishmereni M, Kloft C, Friberg LE (2014) A review of mixed-effects models of tumor growth and effects of anticancer drug treatment used in population analysis. CPT Pharmacometrics Syst Pharmacol 3:12. https://doi.org/10.1038/psp.2014.12

40. Groenland SL, van Nuland M, Bergman AM, de Feijter JM, Dezentje VO, Rosing H, Beijnen JH, Huitema ADR, Steeghs N (2020) Concomitant intake of abiraterone acetate and food to increase pharmacokinetic exposure: real life data from a therapeutic drug monitoring programme. Eur J Cancer 130:32-38. https://doi. org/10.1016/j.ejca.2020.02.012

41. Lacy SA, Miles D, Nguyen L (2017) Clinical pharmacokinetics and pharmacodynamics of cabozantinib. Clin Pharmacokinet 56: 477-491. https://doi.org/10.1007/s40262-016-0461-9

42. Lubberman FJE, Gelderblom H, Hamberg P, Vervenne WL, Mulder SF, Jansman FGA, Colbers A, van der Graaf WTA, Burger DM, Luelmo S, Moes DJAR, van Herpen CML, van Erp NP (2019) The effect of using pazopanib with food vs. fasted on pharmacokinetics, patient safety, and preference (DIET study). Clin Pharmacol Ther 106:1076-1082. https://doi.org/10.1002/cpt.1515

43. Groenland SL, van Eerden RAG, Verheijen RB, de Vries N, Thijssen B, Rosing H, Beijnen JH, Koolen SLW, Mathijssen RHJ, Huitema ADR, Steeghs N (2020) Cost-Neutral Optimization of Pazopanib Exposure by Splitting Intake Moments: A Prospective Pharmacokinetic Study in Cancer Patients. Clin Pharmacokinet. https://doi.org/10.1007/s40262-020-00863-5

44. Lankheet NAG, Desar IME, Mulder SF, Burger DM, Kweekel DM, van Herpen CML, van der Graaf WTA, van Erp NP (2017) Optimizing the dose in cancer patients treated with imatinib, sunitinib and pazopanib. Br J Clin Pharmacol 83:2195-2204. https:// doi.org/10.1111/bcp.13327

45. Larson RA, Druker BJ, Guilhot F, O'Brien SG, Riviere GJ, Krahnke T, Gathmann I, Wang Y (2008) Imatinib 
pharmacokinetics and its correlation with response and safety in chronic-phase chronic myeloid leukemia: a subanalysis of the IRIS study. Blood 111:4022-4028. https://doi.org/10.1182/ blood-2007-10-116475

46. Demetri GD, Wang Y, Wehrle E, Racine A, Nikolova Z, Blanke CD, Joensuu H, Von Mehren M (2009) Imatinib plasma levels are correlated with clinical benefit in patients with unresectable/ metastatic gastrointestinal stromal tumors. J Clin Oncol 27: 3141-3147. https://doi.org/10.1200/jco.2008.20.4818

47. Lankheet NAG, Kloth JSL, Gadellaa-Van Hooijdonk CGM, Cirkel GA, Mathijssen RHJ, Lolkema MPJK, Schellens JHM, Voest EE, Sleijfer S, De Jonge MJA, Haanen JBAG, Beijnen JH, Huitema ADR, Steeghs N (2014) Pharmacokinetically guided sunitinib dosing: a feasibility study in patients with advanced solid tumours. Br J Cancer 110:2441-2449. https://doi.org/10.1038/ bjc.2014.194

48. Verheijen RB, Bins S, Mathijssen RHJ, Lolkema MP, van Doorn L, Schellens JHM, Beijnen JH, Langenberg MHG, Huitema ADR, Steeghs N (2016) Individualized pazopanib dosing: a prospective feasibility study in cancer patients. Clin Cancer Res 22:57385746. https://doi.org/10.1158/1078-0432.ccr-16-1255

49. Groenland SL, Geel DR, Janssen JM, de Vries N, Rosing H, Beijnen JH, Burgers JA, Smit EF, Huitema ADR, Steeghs N (2020) Exposure-Response Analyses of Anaplastic Lymphoma Kinase Inhibitors Crizotinib and Alectinib in Non-Small Cell Lung Cancer Patients. Clin Pharmacol Ther. https://doi.org/10. 1002/cpt.1989

50. Ouellet D, Kassir N, Chiu J, Mouksassi MS, Leonowens C, Cox D, DeMarini DJ, Gardner O, Crist W, Patel K (2016) Population pharmacokinetics and exposure-response of trametinib, a MEK inhibitor, in patients with BRAF V600 mutation-positive melanoma. Cancer Chemother Pharmacol 77:807-817. https://doi.org/10. 1007/s00280-016-2993-y

51. Goldwirt L, Chami I, Feugeas JP, Pages C, Brunet-Possenti F, Allayous C, Baroudjian B, Madelaine I, Sauvageon H, Mourah S, Lebbé C (2016) Reply to "plasma vemurafenib concentrations in advanced BRAFV600mut melanoma patients: impact on tumour response and tolerance" by Funck-Brentano et al. Ann Oncol 27:363-364. https://doi.org/10.1093/annonc/mdv538

52. Funck-Brentano E, Alvarez JC, Longvert C, Abe E, Beauchet A, Funck-Brentano C, Saiag P (2015) Plasma vemurafenib concentrations in advanced BRAFV600mut melanoma patients: impact on tumour response and tolerance. Ann Oncol 26:1470-1475. https://doi.org/10.1093/annonc/mdv189

53. Kramkimel N, Thomas-Schoemann A, Sakji L, Golmard JL, Noe G, Regnier-Rosencher E, Chapuis N, Maubec E, Vidal M, Avril MF, Goldwasser F, Mortier L, Dupin N, Blanchet B (2016) Vemurafenib pharmacokinetics and its correlation with efficacy and safety in outpatients with advanced BRAF-mutated melanoma. Target Oncol 11:59-69. https://doi.org/10.1007/s11523-0150375-8

54. Kichenadasse G, Hughes JH, Miners JO, Mangoni AA, Rowland A, Hopkins AM, Sorich MJ (2019) Relationship between vemurafenib plasma concentrations and survival outcomes in patients with advanced melanoma. Cancer Chemother Pharmacol. https://doi.org/10.1007/s00280-019-04002-1

55. Groenland SL, van Eerden RAG, Verheijen RB, Koolen SLW, Moes DJAR, Desar IME, Reyners AKL, Gelderblom HJ, van Erp NP, Mathijssen RHJ, Huitema ADR, Steeghs N (2019) Therapeutic drug monitoring of oral anticancer drugs: the Dutch Pharmacology Oncology Group-Therapeutic Drug Monitoring Protocol for a Prospective Study. In: Ther Drug Monit 41

56. Food and Drug Administration. Center for Drug Evaluation and Research and Center for Biologics Evaluation and Research. (2003) Guidance for industry - pharmacokinetics in patients with impaired hepatic function - study design, data analysis, and impact on dosing and labelling. Draft Guidance Document. https://www.fda.gov/media/71311/download. Accessed 23 March 2020

57. Food and Drug Administration. Center for Drug Evaluation and Research and Center for Biologics Evaluation and Research. (2010) Guidance for industry - pharmacokinetics in patients with impaired renal function - study design, data analysis, and impact on dosing and labelling. Draft Guidance Document. https://www. fda.gov/media/78573/download. Accessed 23 March 2020

58. Food and Drug Administration. Center for Drug Evaluation and Research and Center for Biologics Evaluation and Research. Pediatric study plans: content of and process for submitting initial pediatric study plans and amended initial pediatric study plans guidance for industry. Accessed 23 March 2020

59. Groenland SL, Van Eerden RAG, Koolen SL, Moes DJAR, Desar IME, Touw DJ, Imholz ALT, Reyners AKL, Gelderblom H, van Erp NP, Mathijssen RHJ, Huitema ADR, Steeghs N (2019) Therapeutic drug monitoring of oral anticancer drugspreliminary results of a prospective study. Ann Oncol 30:442O. https://doi.org/10.1093/annonc/mdz244.004

60. Houk BE, Bello CL, Poland B, Rosen LS, Demetri GD, Motzer RJ (2010) Relationship between exposure to sunitinib and efficacy and tolerability endpoints in patients with cancer: results of a pharmacokinetic/pharmacodynamic meta-analysis. Cancer Chemother Pharmacol 66:357-371. https://doi.org/10.1007/ s00280-009-1170-y

61. Suttle AB, Ball HA, Molimard M, Hutson TE, Carpenter C, Rajagopalan D, Lin Y, Swann S, Amado R, Pandite L (2014) Relationships between pazopanib exposure and clinical safety and efficacy in patients with advanced renal cell carcinoma. Br J Cancer 111:1909-1916. https://doi.org/10.1038/bjc.2014.503

62. Carton E, Noe G, Huillard O, Golmard L, Giroux J, Cessot A, Saidu NEB, Peyromaure M, Zerbib M, Narjoz C, Guibourdenche J, Thomas A, Vidal M, Goldwasser F, Blanchet B, Alexandre J (2017) Relation between plasma trough concentration of abiraterone and prostate-specific antigen response in metastatic castration-resistant prostate cancer patients. Eur J Cancer 72:54 61. https://doi.org/10.1016/j.ejca.2016.11.027

63. van Nuland M, Groenland SL, Bergman AM, Steeghs N, Rosing H, Venekamp N, Huitema ADR, Beijnen JH (2019) Exposureresponse analyses of abiraterone and its metabolites in real-world patients with metastatic castration-resistant prostate cancer. Prostate cancer Prostatic Dis https://doi.org/10.1038/s41391-0190179-5

64. Ravaud A, Urva SR, Grosch K, Cheung WK, Anak O, Sellami DB (2014) Relationship between everolimus exposure and safety and efficacy: meta-analysis of clinical trials in oncology. Eur $\mathrm{J}$ Cancer 50:486-495. https://doi.org/10.1016/j.ejca.2013.11.022

65. Verheijen RB, Atrafi F, Schellens JHM, Beijnen JH, Huitema ADR, Mathijssen RHJ, Steeghs N (2018) Pharmacokinetic optimization of everolimus dosing in oncology: a randomized crossover trial. Clin Pharmacokinet 57:637-644. https://doi.org/10. 1007/s40262-017-0582-9

66. Combes FP, Guillaume C, Neva B, Penny C, William Z, Yin H, Nedelman J (2018) Population pharmacokineticspharmacodynamics of oral everolimus in patients with seizures associated with tuberous sclerosis complex. J Pharmacokinet Pharmacodyn 45:707-719. https://doi.org/10.1007/s10928-0189600-2

67. Guilhot F, Hughes TP, Cortes J, Druker BJ, Baccarani M, Gathmann I, Hayes M, Granvil C, Wang Y, Inserm CIC, Poitiers CHU, De Hospital RA, Inserm P, De CHU (2012) Plasma exposure of imatinib and its correlation with clinical response in the Tyrosine Kinase Inhibitor Optimization and Selectivity Trial. Haematologica 97:731-738. https://doi.org/10. 3324/haematol.2011.045666 
68. Miura M (2015) Therapeutic drug monitoring of imatinib, nilotinib, and dasatinib for patients with chronic myeloid leukemia. Biol Pharm Bull 38:645-654

69. Bouchet S, Poulette S, Titier K, Moore N, Lassalle R, Abouelfath A, Italiano A, Chevreau C, Bompas E, Collard O, Duffaud F, Rios M, Cupissol D, Adenis A, Ray-Coquard I, Bouché O, Le Cesne A, Bui B, Blay JY, Molimard M (2016) Relationship between imatinib trough concentration and outcomes in the treatment of advanced gastrointestinal stromal tumours in a real-life setting. Eur J Cancer 57:31-38. https://doi.org/10.1016/j.ejca.2015.12.029

70. Widmer N, Decosterd LA, Csajka C, Leyvraz S, Duchosal MA, Rosselet A, Rochat B, Eap CB, Henry H, Biollaz J, Buclin T (2006) Population pharmacokinetics of imatinib and the role of $\alpha 1$-acid glycoprotein. Br J Clin Pharmacol 62:97-112. https://doi. org/10.1111/j.1365-2125.2006.02719.x

71. Golabchifar AA, Rezaee S, Ghavamzadeh A, Alimoghaddam K, Dinan NM, Rouini MR (2014) Population pharmacokinetics of imatinib in Iranian patients with chronic-phase chronic myeloid leukemia. Cancer Chemother Pharmacol 74:85-93. https://doi. org/10.1007/s00280-014-2473-1

72. Schmidli H, Capdeville R, Hensley M, Peng B, Gathmann I, Racine-Poon A, Riviere G-J, Bolton AE (2005) Population pharmacokinetics of imatinib mesylate in patients with chronic-phase chronic myeloid leukaemia: results of a phase III study. Br J Clin Pharmacol 60:35-44. https://doi.org/10.1111/j.1365-2125.2005. 02372.x

73. Farag S, Verheijen RB, Martijn Kerst J, Cats A, Huitema ADR, Steeghs N (2017) Imatinib pharmacokinetics in a large observational cohort of gastrointestinal stromal tumour patients. Clin Pharmacokinet 56:287-292. https://doi.org/10.1007/s40262-0160439-7

74. Eechoute K, Fransson MN, Reyners AK, De Jong FA, Sparreboom A, Van Der Graaf WTA, Friberg LE, Schiavon G, Wiemer EAC, Verweij J, Loos WJ, Mathijssen RHJ, De Giorgi U (2012) A long-term prospective population pharmacokinetic study on imatinib plasma concentrations in GIST patients. Clin Cancer Res 18:5780-5787. https://doi.org/10.1158/1078-0432. ccr-12-0490

75. Sternberg CN, Donskov F, Haas NB, Doehn C, Russo P, Elmeliegy M, Baneyx G, Banerjee H, Aimone P, Motzer RJ (2018) Pazopanib exposure relationship with clinical efficacy and safety in the adjuvant treatment of advanced renal cell carcinoma. Clin Cancer Res 24:3005-3013. https://doi.org/10.1158/ 1078-0432.ccr-17-2652

76. Verheijen RB, Swart LE, Beijnen JH, Schellens JHM, Huitema ADR, Steeghs N (2017) Exposure-survival analyses of pazopanib in renal cell carcinoma and soft tissue sarcoma patients: opportunities for dose optimization. Cancer Chemother Pharmacol 80: 1171-1178. https://doi.org/10.1007/s00280-017-3463-x

77. Noda S, Yoshida T, Hira D, Murai R, Tomita K, Tsuru T, Kageyama S, Kawauchi A, Ikeda Y, Morita S y, Terada T (2018) Exploratory investigation of target pazopanib concentration range for patients with renal cell carcinoma. Clin Genitourin Cancer. https://doi.org/10.1016/j.clgc.2018.12.001

78. Yu H, van Erp N, Bins S, Mathijssen RHJ, Schellens JHM, Beijnen JH, Steeghs N, Huitema ADR (2017) Development of a pharmacokinetic model to describe the complex pharmacokinetics of pazopanib in cancer patients. Clin Pharmacokinet 56:293-303. https://doi.org/10.1007/s40262-016-0443-y

79. Noda S, Otsuji T, Baba M, Yoshida T, Kageyama S, Okamoto K, Okada Y, Kawauchi A, Onishi H, Hira D, Morita SY, Terada T (2015) Assessment of Sunitinib-Induced Toxicities and Clinical Outcomes Based on Therapeutic Drug Monitoring of Sunitinib for Patients with Renal Cell Carcinoma. Clin Genitourin Cancer 13: 350-358. https://doi.org/10.1016/j.clgc.2015.01.007
80. Faivre S, Delbaldo C, Vera K, Robert C, Lozahic S, Lassau N, Bello C, Deprimo S, Brega N, Massimini G, Armand JP, Scigalla P, Raymond E (2006) Safety, pharmacokinetic, and antitumor activity of SU11248, a novel oral multitarget tyrosine kinase inhibitor, in patients with cancer. J Clin Oncol 24:25-35. https://doi. org/10.1200/jco.2005.02.2194

81. Khosravan R, Motzer RJ, Fumagalli E, Rini BI (2016) Population Pharmacokinetic/Pharmacodynamic Modeling of Sunitinib by Dosing Schedule in Patients with Advanced Renal Cell Carcinoma or Gastrointestinal Stromal Tumor. Clin Pharmacokinet 55:1251-1269. https://doi.org/10.1007/s40262016-0404-5

82. Diekstra MH, Fritsch A, Kanefendt F, Swen JJ, Djar M, Sörgel F, Kinzig M, Stelzer C, Schindele D, Gauler T, Hauser S, Houtsma D, Roessler M, Moritz B, Mross K, Bergmann L, Oosterwijk E, Kiemeney LA, Guchelaar HJ, Jaehde U (2017) Population modeling integrating pharmacokinetics, pharmacodynamics, pharmacogenetics, and clinical outcome in patients with sunitinib-treated cancer. CPT Pharmacometrics Syst Pharmacol 6:604-613. https://doi.org/10.1002/psp4.12210

83. Madlensky L, Natarajan L, Tchu S, Pu M, Mortimer J, Flatt SW, Nikoloff DM, Hillman G, Fontecha MR, Lawrence HJ, Parker BA, Wu AHB, Pierce JP (2011) Tamoxifen metabolite concentrations, CYP2D6 genotype, and breast cancer outcomes. Clin Pharmacol Ther 89:718-725. https://doi.org/10.1038/clpt.2011.32

84. Saladores P, Mürdter T, Eccles D, Chowbay B, Zgheib NK, Winter S, Ganchev B, Eccles B, Gerty S, Tfayli A, Lim JSL, Yap YS, Ng RCH, Wong NS, Dent R, Habbal MZ, Schaeffeler E, Eichelbaum M, Schroth W, Schwab M, Brauch H (2015) Tamoxifen metabolism predicts drug concentrations and outcome in premenopausal patients with early breast cancer. Pharm J 15: 84-94. https://doi.org/10.1038/tpj.2014.34

85. Mueller-Schoell A, Klopp-schulze L, Schroth W, Michelet R, Brauch H, Huisinga W, Joerger M, Neven P, Koolen SLW, Mathijssen RHJ, Copson E, Eccles D, Chen S, Chowbay B, Tfayli A, Zgheib NK, Schwab M, Kloft C (2020) Obesity alters endoxifen plasma levels in young breast cancer patients: A pharmacometric simulation approach. Clin Pharmacol Ther. https://doi.org/10.1002/cpt.1960

86. Pistilli B, Paci A, Ferreira AR, Di Meglio A, Poinsignon V, Bardet A, Menvielle G, Dumas A, Pinto S, Dauchy S, Fasse L, Cottu PH, Lerebours F, Coutant C, Lesur A, Tredan O, Soulie P, Vanlemmens L, Jouannaud C, Levy C, Everhard S, Arveux P, Martin AL, Dima A, Lin NU, Partridge AH, Delaloge S, Michiels S, André F, Vaz-Luis I (2020) Serum Detection of Nonadherence to Adjuvant Tamoxifen and Breast Cancer Recurrence Risk. J Clin Oncol. https://doi.org/10.1200/jco.19. 01758

87. Welzen MEB, Dezentjé VO, van Schaik RHN, Colbers APH, Guchelaar H-J, van Erp NP, den Hartigh J, Burger DM, van Laarhoven HWM (2015) The Effect of Tamoxifen Dose Increment in Patients With Impaired CYP2D6 Activity. Ther Drug Monit 37:501-507. https://doi.org/10.1097/ftd. 0000000000000195

88. Barginear MF, Jaremko M, Peter I, Yu C, Kasai Y, Kemeny M, Raptis G, Desnick RJ (2011) Increasing tamoxifen dose in breast cancer patients based on CYP2D6 genotypes and endoxifen levels: Effect on active metabolite isomers and the antiestrogenic activity score. Clin Pharmacol Ther 90:605-611. https://doi.org/ 10.1038/clpt.2011.153

89. Dezentjé VO, Opdam FL, Gelderblom H, Hartigh d J, Van der Straaten T, Vree R, Maartense E, Smorenburg CH, Putter H, Dieudonné AS, Neven P, Van de Velde CJH, Nortier JWR, Guchelaar HJ (2015) CYP2D6 genotype- and endoxifen-guided tamoxifen dose escalation increases endoxifen serum 
concentrations without increasing side effects. Breast Cancer Res Treat 153:583-590. https://doi.org/10.1007/s10549-015-3562-5

90. Irvin WJ, Walko CM, Weck KE, Ibrahim JG, Chiu WK, Dees EC, Moore SG, Olajide OA, Graham ML, Canale ST, Raab RE, Corso SW, Peppercorn JM, Anderson SM, Friedman KJ, Ogburn ET, Desta Z, Flockhart DA, McLeod HL, Evans JP, Carey LA (2011) Genotype-Guided Tamoxifen Dosing Increases Active Metabolite Exposure in Women With Reduced CYP2D6 Metabolism: A Multicenter Study. J Clin Oncol 29:3232-3239. https://doi.org/ $10.1200 /$ jco.2010.31.4427

91. Khalaj Z, Baratieh Z, Nikpour P, Schwab M, Schaeffeler E, Mokarian F, Khanahmad H, Salehi R, Mürdter TE, Salehi M (2019) Clinical Trial: CYP2D6 Related Dose Escalation of Tamoxifen in Breast Cancer Patients With Iranian Ethnic Background Resulted in Increased Concentrations of Tamoxifen and Its Metabolites. Front Pharmacol 10:1-13. https://doi.org/10. 3389/fphar.2019.00530

92. Klopp-Schulze L, Mueller-Schoell A, Neven P, Koolen SL, Mathijssen R, Joerger M, Kloft C (2020) Integrated data analysis of six clinical studies points toward model-informed precision dosing of tamoxifen. Front Pharmacol 11:1-19. https://doi.org/ 10.3389/fphar.2020.00283

93. Herbrink M, de Vries N, Rosing H, Huitema ADR, Nuijen B, Schellens JHM, Beijnen JH (2016) Quantification of 11 Therapeutic Kinase Inhibitors in Human Plasma for Therapeutic Drug Monitoring Using Liquid Chromatography Coupled With Tandem Mass Spectrometry. Ther Drug Monit 38:649-656. https://doi.org/10.1097/ftd.0000000000000349

94. Herbrink M, de Vries N, Rosing H, Huitema ADR, Nuijen B, Schellens JHM, Beijnen JH (2018) Development and validation of a liquid chromatography-tandem mass spectrometry analytical method for the therapeutic drug monitoring of eight novel anticancer drugs. Biomed Chromatogr 32:1-9. https://doi.org/10.1002/ bmc. 4147

95. Janssen JM, de Vries N, Venekamp N, Rosing H, Huitema ADR, Beijnen JH (2019) Development and validation of a liquid chromatography-tandem mass spectrometry assay for nine oral anticancer drugs in human plasma. J Pharm Biomed Anal 174:561566. https://doi.org/10.1016/j.jpba.2019.06.034

96. van Nuland M, Venekamp N, de Vries N, de Jong KAM, Rosing H, Beijnen JH (2019) Development and validation of an UPLCMS/MS method for the therapeutic drug monitoring of oral antihormonal drugs in oncology. J Chromatogr B 1106-1107:26-34. https://doi.org/10.1016/j.jchromb.2019.01.001

97. Kok MGM, Fillet M (2018) Volumetric absorptive microsampling: Current advances and applications. J Pharm Biomed Anal 147:288-296. https://doi.org/10.1016/j.jpba.2017. 07.029

98. Protti M, Mandrioli R, Mercolini L (2019) Tutorial: Volumetric absorptive microsampling (VAMS). Anal Chim Acta 1046:3247. https://doi.org/10.1016/j.aca.2018.09.004

99. Stuyckens K, Saad F, Xu XS, Ryan CJ, Smith MR, Griffin TW, Yu MK, Vermeulen A, Nandy P, Poggesi I (2014) Population Pharmacokinetic Analysis of Abiraterone in ChemotherapyNaïve and Docetaxel-Treated Patients with Metastatic Castration-Resistant Prostate Cancer. Clin Pharmacokinet 53: 1149-1160. https://doi.org/10.1007/s40262-014-0178-6

100. Szmulewitz RZ, Peer C, Ibraheem A, Martinez E, Kozloff MF, Carthon B, Donald Harvey R, Fishkin P, Yong WP, Chiong E, Nabhan C, Karrison T, Figg WD, Stadler WM, Ratain MJ (2018) Prospective International Randomized Phase II Study of LowDose Abiraterone With Food Versus Standard Dose Abiraterone In Castration-Resistant Prostate Cancer. J Clin Oncol 36:13891395. https://doi.org/10.1200/jco.2017.76.4381

101. Rini BI, Garrett M, Poland B, Dutcher JP, Rixe O, Wilding G, Stadler WM, Pithavala YK, Kim S, Tarazi J, Motzer RJ (2013)
Axitinib in metastatic renal cell carcinoma: Results of a pharmacokinetic and pharmacodynamic analysis. J Clin Pharmacol 53: 491-504. https://doi.org/10.1002/jcph.73

102. Tsuchiya N, Igarashi R, Suzuki-Honma N, Fujiyama N, Narita S, Inoue T, Saito M, Akihama S, Tsuruta H, Miura M, Habuchi T (2015) Association of pharmacokinetics of axitinib with treatment outcome and adverse events in advanced renal cell carcinoma patients. J Clin Oncol 33:506. https://doi.org/10.1200/jco.2015. 33.7_suppl.506

103. Committee for Medicinal Products for Human Use (CHMP) European Medicines Agency (2012) Inlyta European public assessment report. https://www.ema.europa.eu/documents/productinformation/inlyta-epar-product-information_en.pdf. Accessed 17 June 2019

104. Food and Drug Administration. Center for Drug Evaluation and Research (2012) Axitinib Clinical Pharmacology and Biopharmaceutics Review. https://www.accessdata.fda.gov/ drugsatfda docs/nda/2012/202324Orig1s000ClinPharmR.pdf. Accessed $1 \overline{8}$ June 2019

105. Rini BI, Melichar B, Fishman MN, Oya M, Pithavala YK, Chen Y, Bair AH, Grünwald V (2015) Axitinib dose titration: Analyses of exposure, blood pressure and clinical response from a randomized phase II study in metastatic renal cell carcinoma. Ann Oncol 26:1372-1377. https://doi.org/10.1093/annonc/mdv103

106. Rini BI, Melichar B, Ueda T, Grünwald V, Fishman MN, Arranz JA, Bair AH, Pithavala YK, Andrews GI, Pavlov D, Kim S, Jonasch E (2013) Axitinib with or without dose titration for firstline metastatic renal-cell carcinoma: A randomised double-blind phase 2 trial. Lancet Oncol 14:1233-1242. https://doi.org/10. 1016/s1470-2045(13)70464-9

107. Thiery-Vuillemin A, Mouillet G, Nguyen Tan Hon T, Montcuquet P, Maurina T, Almotlak H, Stein U, Montange D, Foubert A, Nerich V, Pivot X, Royer B (2014) Impact of everolimus blood concentration on its anti-cancer activity in patients with metastatic renal cell carcinoma. Cancer Chemother Pharmacol 73:999-1007. https://doi.org/10.1007/s00280-014-2435-7

108. de Wit D, Schneider TC, Moes DJAR, Roozen CFM, den Hartigh J, Gelderblom H, Guchelaar HJ, van der Hoeven JJ, Links TP, Kapiteijn E, van Erp NP (2016) Everolimus pharmacokinetics and its exposure-toxicity relationship in patients with thyroid cancer. Cancer Chemother Pharmacol 78:63-71. https://doi.org/10. 1007/s00280-016-3050-6

109. ter Heine R, van Erp NP, Guchelaar HJ, de Fijter JW, Reinders MEJ, van Herpen CM, Burger DM, Moes DJAR (2018) A pharmacological rationale for improved everolimus dosing in oncology and transplant patients. Br J Clin Pharmacol 84:1575-1586. https://doi.org/10.1111/bcp.13591

110. Krueger DA, Care MM, Holland K, Agricola K, Tudor C, Mangeshkar P, Wilson KA, Byars A, Sahmoud T, Franz DN (2010) Everolimus for subependymal giant-cell astrocytomas in tuberous sclerosis. N Engl J Med 363:1801-1811. https://doi.org/ 10.1056/nejmoa1001671

111. Shipkova M, Hesselink DA, Holt DW, Billaud EM, Van Gelder T, Kunicki PK, Brunet M, Budde K, Barten MJ, De Simone P, Wieland E, Lopez OM, Masuda S, Seger C, Picard N, Oellerich M, Langman LJ, Wallemacq P, Morris RG, Thompson C, Marquet P (2016) Therapeutic drug monitoring of everolimus: A consensus report. Ther Drug Monit 38:143-169. https://doi.org/ $10.1097 / \mathrm{ftd} .0000000000000260$

112. Kobayashi H, Sato K, Niioka T, Miura H, Ito H, Miura M (2015) Relationship among gefitinib exposure, polymorphisms of its metabolizing enzymes and transporters, and side effects in Japanese patients with non-small-cell lung cancer. Clin Lung Cancer 16: 274-281. https://doi.org/10.1016/j.cllc.2014.12.004

113. Perez CA, Song H, Raez LE, Agulnik M, Grushko TA, Dekker A, Stenson K, Blair EA, Olopade OI, Seiwert TY, Vokes EE, Cohen 
EEW (2012) Phase II study of gefitinib adaptive dose escalation to skin toxicity in recurrent or metastatic squamous cell carcinoma of the head and neck. Oral Oncol 48:887-892. https://doi.org/10. 1016/j.oraloncology.2012.03.020

114. Picard S, Titier K, Etienne G, Teilhet E, Ducint D, Bernard MA, Lassalle R, Marit G, Reiffers J, Begaud B, Moore N, Molimard M, Mahon FX (2007) Trough imatinib plasma levels are associated with both cytogenetic and molecular responses to standard-dose imatinib in chronic myeloid leukemia. Blood 109:3496-3499. https://doi.org/10.1182/blood-2006-07-036012

115. Lankheet NAG, Knapen LM, Schellens JHM, Beijnen JH, Steeghs N, Huitema ADR (2014) Plasma concentrations of tyrosine kinase inhibitors imatinib, erlotinib, and sunitinib in routine clinical outpatient cancer care. Ther Drug Monit 36:326-334. https://doi.org/10.1097/ftd.0000000000000004

116. Widmer N, Decosterd LA, Leyvraz S, Duchosal MA, Rosselet A, Debiec-Rychter M, Csajka C, Biollaz J, Buclin T (2008) Relationship of imatinib-free plasma levels and target genotype with efficacy and tolerability. Br J Cancer 98:1633-1640. https:// doi.org/10.1038/sj.bjc.6604355

117. Delbaldo C, Chatelut E, Ré M, Deroussent A, Séronie-Vivien S, Jambu A, Berthaud P, Le Cesne A, Blay JY, Vassal G (2006) Pharmacokinetic-pharmacodynamic relationships of imatinib and its main metabolite in patients with advanced gastrointestinal stromal tumors. Clin Cancer Res 12:6073-6078. https://doi.org/10. 1158/1078-0432.ccr-05-2596

118. Heath EI, Chiorean EG, Sweeney CJ, Hodge JP, Lager JJ, Forman K, Malburg L, Arumugham T, Dar MM, Suttle AB, Gainer SD, Lorusso P (2010) A phase I study of the pharmacokinetic and safety profiles of oral pazopanib with a high-fat or low-fat meal in patients with advanced solid tumors. Clin Pharmacol Ther 88: 818-823. https://doi.org/10.1038/clpt.2010.199

119. Lankheet NAG, Kloth JSL, Gadellaa-Van Hooijdonk CGM, Cirkel GA, Mathijssen RHJ, Lolkema MPJK, Schellens JHM, Voest EE, Sleijfer S, De Jonge MJA, Haanen JBAG, Beijnen JH, Huitema ADR, Steeghs N (2014) Pharmacokinetically guided sunitinib dosing: A feasibility study in patients with advanced solid tumours. Br J Cancer 110:2441-2449. https://doi.org/10. 1038/bjc.2014.194

120. Teo YL, Chue XP, Chau NM, Tan MH, Kanesvaran R, Wee HL, Ho HK, Chan A (2015) Association of drug exposure with toxicity and clinical response in metastatic renal cell carcinoma patients receiving an attenuated dosing regimen of sunitinib. Target Oncol 10:429-437. https://doi.org/10.1007/s11523-014-0349-2

121. Takasaki S, Kawasaki Y, Kikuchi M, Tanaka M, Suzuka M, Noda A, Sato Y, Yamashita S, Mitsuzuka K, Saito H, Ito A, Yamaguchi H, Arai Y, Mano N (2018) Relationships between sunitinib plasma concentration and clinical outcomes in Japanese patients with metastatic renal cell carcinoma. Int J Clin Oncol 23:936-943. https://doi.org/10.1007/s10147-018-1302-7

122. Jager NGL, Rosing H, Schellens JHM, Linn SC, Beijnen JH (2014) Tamoxifen dose and serum concentrations of tamoxifen and six of its metabolites in routine clinical outpatient care. Breast Cancer Res Treat 143:477-483. https://doi.org/10.1007/ s10549-013-2826-1

123. Flaherty KT, Robert C, Hersey P, Nathan P, Garbe C, Milhem M, Demidov LV, Hassel JC, Rutkowski P, Mohr P, Dummer R, Trefzer U, Larkin JMG, Utikal J, Dreno B, Nyakas M, Middleton MR, Becker JC, Casey M, Sherman LJ, Wu FS, Ouellet D, Martin A-M, Patel K, Schadendorf D (2012) Improved Survival with MEK Inhibition in BRAF-Mutated Melanoma. N Engl J Med 367:107-114. https://doi.org/10.1056/ nejmoa1203421

124. Food and Drug Administration. Center for Drug Evaluation and Research (2013) Trametinib Clinical Pharmacology and Biopharmaceutics Review. https://www.accessdata.fda.gov/
drugsatfda_docs/nda/2013/204114Orig1s000ClinPharmR.pdf. Accessed 24 March 2020

125. Food and Drug Administration. Center for Drug Evaluation and Research (2015) Alectinib Clinical Pharmacology and Biopharmaceutics Review. https://www.accessdata.fda.gov/ drugsatfda_docs/nda/2015/208434Orig1s000ClinPharmR.pdf. Accessed 07 April 2019

126. Committee for Medicinal Products for Human Use European Medicines Agency (2017) Alecensa European public assessment report. https://www.ema.europa.eu/en/documents/productinformation/alecensa-epar-product-information_en.pdf. Accessed 07 April 2019

127. Morcos PN, Nueesch E, Jaminion F, Guerini E, Hsu JC, Bordogna W, Balas B, Mercier F (2018) Exposure-response analysis of alectinib in crizotinib-resistant ALK-positive non-small cell lung cancer. Cancer Chemother Pharmacol 82:129-138. https://doi. org/10.1007/s00280-018-3597-5

128. Food and Drug Administration. Center for Drug Evaluation and Research (2011) Crizotinib Clinical Pharmacology and Biopharmaceutics Review. http://www.accessdata.fda.gov/ drugsatfda_docs/nda/2011/202570Orig1s000ClinPharmR.pdf. Accessed 25 Mar 2019

129. Kurata Y, Miyauchi N, Suno M, Ito T, Sendo T, Kiura K (2015) Correlation of plasma crizotinib trough concentration with adverse events in patients with anaplastic lymphoma kinase positive nonsmall-cell lung cancer. J Pharm Heal Care Sci 1:8. https://doi.org/ 10.1186/s40780-014-0008-x

130. Wang E, Nickens DJ, Bello A, Khosravan R, Amantea M, Wilner KD, Parivar K, Tan W (2016) Clinical implications of the pharmacokinetics of crizotinib in populations of patients with nonsmall cell lung cancer. Clin Cancer Res 22:5722-5728. https:// doi.org/10.1158/1078-0432.ccr-16-0536

131. Hidalgo M, Siu LL, Nemunaitis J, Rizzo J, Hammond LA, Takimoto C, Eckhardt SG, Tolcher A, Britten CD, Denis L, Ferrante K, Von Hoff DD, Silberman S, Rowinsky EK (2001) Phase I and pharmacologic study of OSI-774, an epidermal growth factor receptor tyrosine kinase inhibitor, in patients with advanced solid malignancies. J Clin Oncol 19:3267-3279. https:// doi.org/10.1200/jco.2001.19.13.3267

132. Soulieres D, Senzer NN, Vokes EE, Hidalgo M, Agarvala SS, Siu LL (2004) Multicenter phase II study of erlotinib, an oral epidermal growth factor receptor tyrosine kinase inhibitor, in patients with recurrent or metastatic squamous cell cancer of the head and neck. J Clin Oncol 22:77-85. https://doi.org/10.1200/jco. 2004.06.075

133. Tiseo M, Andreoli R, Gelsomino F, Mozzoni P, Azzoni C, Bartolotti M, Bortesi B, Goldoni M, Silini EM, De Palma G, Mutti A, Ardizzoni A (2014) Correlation between erlotinib pharmacokinetics, cutaneous toxicity and clinical outcomes in patients with advanced non-small cell lung cancer (NSCLC). Lung Cancer 83:265-271. https://doi.org/10.1016/j.lungcan.2013.12.001

134. Lu JF, Eppler SM, Wolf J, Hamilton M, Rakhit A, Bruno R, Lum BL (2006) Clinical pharmacokinetics of erlotinib in patients with solid tumors and exposure-safety relationship in patients with nonsmall cell lung cancer. Clin Pharmacol Ther 80:136-145. https:// doi.org/10.1016/j.clpt.2006.04.007

135. Endo-Tsukude C, Sasaki J, Saeki S, Iwamoto N, Inaba M, Ushijima S, Kishi H, Fujii S, Semba H, Kashiwabara K, Tsubata Y, Hayashi M, Kai Y, Saito H, Isobe T, Kohrogi H, Hamada A (2018) Population Pharmacokinetics and Adverse Events of Erlotinib in Japanese Patients with Non-small-cell Lung Cancer: Impact of Genetic Polymorphisms in Metabolizing Enzymes and Transporters. Biol Pharm Bull 41: 47-56. https://doi.org/10.1248/bpb.b17-00521

136. Food and Drug Administration. Center for Drug Evaluation and Research (2018) NDA Multi-Disciplinary Review and Evaluation 
NDA 211349 Xospata (gilteritinib). https://www.accessdata.fda. gov/d r u g s a t f d a d o c s / n d a / $2018 /$ 211349Orig1s000MultidisciplineR.pdf. Accessed 13 February 2020

137. Food and Drug Administration. Center for Drug Evaluation and Research (1997) Letrozole Clinical Pharmacology and Biopharmaceutics Review. https://www.accessdata.fda.gov/ drugsatfda docs/nda/97/20726 FEMARA2.5MG BIOPHARMR.PDF. Accessed 14 February 2020

138. Desta Z, Kreutz Y, Nguyen AT, Li L, Skaar T, Kamdem LK, Henry NL, Hayes DF, Storniolo AM, Stearns V, Hoffmann E, Tyndale RF, Flockhart DA (2011) Plasma letrozole concentrations in postmenopausal women with breast cancer are associated with CYP2A6 genetic variants, body mass index, and age. Clin Pharmacol Ther 90:693-700. https://doi.org/10.1038/clpt.2011. 174

139. Tanii H, Shitara Y, Horie T (2011) Population pharmacokinetic analysis of letrozole in Japanese postmenopausal women. Eur J Clin Pharmacol 67:1017. https://doi.org/10.1007/s00228-0111042-3

140. Giles FJ, Yin OQP, Sallas WM, Le Coutre PD, Woodman RC, Ottmann OG, Baccarani M, Kantarjian HM (2013) Nilotinib population pharmacokinetics and exposure-response analysis in patients with imatinib-resistant or -intolerant chronic myeloid leukemia. Eur J Clin Pharmacol 69:813-823. https://doi.org/10.1007/ s00228-012-1385-4

141. Larson RA, Yin OQP, Hochhaus A, Saglio G, Clark RE, Nakamae H, Gallagher NJ, Demirhan E, Hughes TP, Kantarjian HM, Le Coutre PD (2012) Population pharmacokinetic and exposure-response analysis of nilotinib in patients with newly diagnosed $\mathrm{Ph}+$ chronic myeloid leukemia in chronic phase. Eur J Clin Pharmacol 68:723-733. https://doi.org/10.1007/s00228-011-1200-7

142. Takahashi N, Abumiya M, Miura M, Niioka T, Sawada K, Tagawa H, Kameoka Y, Fujishima N (2014) Influence of UGT1A1 *6, *27, and *28 Polymorphisms on Nilotinib-induced Hyperbilirubinemia in Japanese Patients with Chronic Myeloid Leukemia. Drug Metab Pharmacokinet 29:449-454. https://doi. org/10.2133/dmpk.dmpk-14-rg-031

143. Nakahara R, Sumimoto T, Ogata M, Sato Y, Itoh H (2019) Successful determination of nilotinib dosage by therapeutic drug monitoring in a patient with chronic myeloid leukemia developing hepatic dysfunction: A case report. Clin Case Reports 7:14191421. https://doi.org/10.1002/ccr3.2191

144. Nijenhuis CM, Huitema ADR, Blank C, Haanen JBAG, van Thienen JV, Rosing H, Schellens JHM, Beijnen JH (2017) Clinical Pharmacokinetics of Vemurafenib in BRAF-Mutated Melanoma Patients. J Clin Pharmacol 57:125-128. https://doi. org $/ 10.1002 / \mathrm{jcph} .788$

145. Nijenhuis CM, Huitema ADR, Marchetti S, Blank C, Haanen JBAG, van Thienen JV, Rosing H, Schellens JHM, Beijnen JH (2016) The Use of Dried Blood Spots for Pharmacokinetic Monitoring of Vemurafenib Treatment in Melanoma Patients. J Clin Pharmacol. https://doi.org/10.1002/jcph. 728

146. Committee for Medicinal Products for Human Use (CHMP) European Medicines Agency (2012) Zelboraf European public assessment report. https:/www.ema.europa.eu/en/documents/ assessment-report/zelboraf-epar-public-assessment-report_en. pdf. Accessed 24 March 2020

147. Food and Drug Administration. Center for Drug Evaluation and Research (2017) Vemurafenib Clinical Pharmacology and Biopharmaceutics Review. https://www.accessdata.fda.gov/ drugsatfda_docs/nda/2017/202429Orig1s016ClinPharmR.pdf. Accessed 24 March 2020

148. Food and Drug Administration. Center for Drug Evaluation and Research (2018) Abemaciclib Clinical Pharmacology and Biopharmaceutics Review. https://www.accessdata.fda.gov/
drugsatfda_docs/nda/2018/208855Orig1s000MultidisciplineR. pdf. Accessed 12 February 2020

149. Tate SC, Sykes AK, Kulanthaivel P, Chan EM, Turner PK, Cronier DM (2018) A Population Pharmacokinetic and Pharmacodynamic Analysis of Abemaciclib in a Phase I Clinical Trial in Cancer Patients. Clin Pharmacokinet 57:335-344. https:// doi.org/10.1007/s40262-017-0559-8

150. Food and Drug Administration. Center for Drug Evaluation and Research (2012) Afatinib Clinical Pharmacology and Biopharmaceutics Review. https://www.accessdata.fda.gov/ drugsatfda_docs/nda/2013/201292Orig1s000ClinPharmR.pdf. Accessed 12 February 2020

151. Nakao K, Kobuchi S, Marutani S, Iwazaki A, Tamiya A, Isa S, Okishio K, Kanazu M, Tamiya M, Hirashima T, Imai K, Sakaeda $\mathrm{T}$, Atagi S (2019) Population pharmacokinetics of afatinib and exposure-safety relationships in Japanese patients with EGFR mutation-positive non-small cell lung cancer. Sci Rep 9:1-11. https:// doi.org/10.1038/s41598-019-54804-9

152. Lim CK, Wei YF, Tsai MS, Chen KY, Shih JY, Yu CJ (2018) Treatment effectiveness and tolerability of afatinib at different doses in patients with EGFR-mutated lung adenocarcinoma: How low can we go? Eur J Cancer 103:32-40. https://doi.org/ 10.1016/j.ejca.2018.07.128

153. Freiwald M, Schmid U, Fleury A, Wind S, Stopfer P, Staab A (2014) Population pharmacokinetics of afatinib, an irreversible ErbB family blocker, in patients with various solid tumors. Cancer Chemother Pharmacol 73:759-770. https://doi.org/10. 1007/s00280-014-2403-2

154. Food and Drug Administration. Center for Drug Evaluation and Research (2019) NDA/BLA Multi-Disciplinary Review and Evaluation NDA 212526 PIQRAY (alpelisib). https://www. accessdata.fda.gov/drugsatfda docs/nda/2019/ 212526Orig1s000MultidisciplineR.pdf. Accessed 06 April 2020

155. De Buck SS, Jakab A, Boehm M, Bootle D, Juric D, Quadt C, Goggin TK (2014) Population pharmacokinetics and pharmacodynamics of BYL719, a phosphoinositide 3-kinase antagonist, in adult patients with advanced solid malignancies. Br J Clin Pharmacol 78:543-555. https://doi.org/10.1111/bcp.12378

156. Ingle JN, Kalari KR, Buzdar AU, Robson ME, Goetz MP, Desta Z, Barman P, Dudenkov TT, Northfelt DW, Perez EA, Flockhart DA, Williard CV, Wang L, Weinshilboum RM (2015) Estrogens and their precursors in postmenopausal women with early breast cancer receiving anastrozole. Steroids 99:32-38. https://doi.org/ 10.1016/j.steroids.2014.08.007

157. Food and Drug Administration. Center for Drug Evaluation and Research (2018) NDA/BLA Multi-Disciplinary Review and Evaluation NDA 210951 Erleada (apalutamide). https://www. accessdata.fda.gov/drugsatfda_docs/nda/2018/ 210951Orig1s000MultidisciplineR.pdf. Accessed 13 February 2020

158. Pérez-Ruixo C, Pérez-Blanco JS, Chien C, Yu M, Ouellet D, Pérez-Ruixo JJ, Ackaert O (2019) Population Pharmacokinetics of Apalutamide and its Active Metabolite N-DesmethylApalutamide in Healthy and Castration-Resistant Prostate Cancer Subjects. Clin Pharmacokinet 59:229-244. https://doi. org/10.1007/s40262-019-00808-7

159. Food and Drug Administration. Center for Drug Evaluation and Research (2020) NDA/BLA Multi-Disciplinary Review and Evaluation NDA 212608 AYVAKIT (avapritinib). https://www. accessdata.fda.gov/drugsatfda_docs/nda/2020/ 212608Orig1s000MultidisciplineR.pdf. Accessed 06 April 2020

160. Food and Drug Administration. Center for Drug Evaluation and Research (2018) NDA Multi-Disciplinary Review and Evaluation NDA 210498 MEKTOVI $^{\mathrm{TM}}$ (binimetinib). https://www. accessdata.fda.gov/drugsatfda_docs/nda/2018/ 210498Orig1s000MultidisciplineR.pdf. Accessed 13 February 2020 
161. Wollenberg L, Marchand M, Merdjan H, Litwiler K (2018) Development of a Population Pharmacokinetic Model for Binimetinib with Subsequent Exposure-Response Analyses in NRAS Mutant Melanoma. Am Conf Pharmacometrics

162. Hsyu P-H, Mould DR, Abbas R, Amantea M (2014) Population Pharmacokinetic and Pharmacodynamic Analysis of Bosutinib. Drug Metab Pharmacokinet 29:441-448. https://doi.org/10.2133/ dmpk.dmpk-13-rg-126

163. Abbas R, Hsyu PH (2016) Clinical Pharmacokinetics and Pharmacodynamics of Bosutinib. Clin Pharmacokinet 55:11911204. https://doi.org/10.1007/s40262-016-0391-6

164. Hsyu PH, Mould DR, Upton RN, Amantea M (2013) Pharmacokinetic-pharmacodynamic relationship of bosutinib in patients with chronic phase chronic myeloid leukemia. Cancer Chemother Pharmacol 71:209-218. https://doi.org/10.1007/ s00280-012-1998-4

165. Food and Drug Administration. Center for Drug Evaluation and Research (2017) NDA Multi-Disciplinary Review and Evaluation NDA 208772 ALUNBRIG (brigatinib). https:// www.accessdata.fda.gov/drugsatfda_docs/nda/2017/ 208772Orig1s000MultidisciplineR.pdf. Accessed 12 February 2020

166. Lacy S, Nielsen J, Yang B, Miles D, Nguyen L, Hutmacher M (2018) Population exposure-response analysis of cabozantinib efficacy and safety endpoints in patients with renal cell carcinoma. Cancer Chemother Pharmacol 81:1061-1070. https://doi.org/10. 1007/s00280-018-3579-7

167. Nguyen L, Chapel S, Duy B, Lacy S (2019) Cabozantinib exposure - response analyses of efficacy and safety in patients with advanced hepatocellular carcinoma. J Pharmacokinet Pharmacodyn 0123456789. https://doi.org/10.1007/s10928-01909659-y

168. Nguyen L, Chapel S, Tran BD, Lacy S (2019) Updated Population Pharmacokinetic Model of Cabozantinib Integrating Various Cancer Types Including Hepatocellular Carcinoma. J Clin Pharmacol 59:1551-1561. https://doi.org/10.1002/jcph.1467

169. Food and Drug Administration. Center for Drug Evaluation and Research (2014) Ceritinib Clinical Pharmacology and Biopharmaceutics Review. https://www.accessdata.fda.gov/ drugsatfda_docs/nda/2014/205755Orig1s000ClinPharmR.pdf. Accessed 13 February 2020

170. Committee for Medicinal Products for Human Use (CHMP) European Medicines Agency (2015) Ceritinib European Public Assessment report. https://www.ema.europa.eu/en/documents/ assessment-report/zykadia-epar-public-assessment-report_en.pdf. Accessed 13 February 2020

171. Hong Y, Passos VQ, Huang PH, Lau YY (2017) Population Pharmacokinetics of Ceritinib in Adult Patients With Tumors Characterized by Genetic Abnormalities in Anaplastic Lymphoma Kinase. J Clin Pharmacol 57:652-662. https://doi. org/10.1002/jcph.849

172. Ouellet D, Gibiansky E, Leonowens C, O'Hagan A, Haney P, Switzky J, Goodman VL (2014) Population pharmacokinetics of dabrafenib, a BRAF inhibitor: Effect of dose, time, covariates, and relationship with its metabolites. J Clin Pharmacol 54:696-706. https://doi.org/10.1002/jcph.263

173. Food and Drug Administration. Center for Drug Evaluation and Research (2013) Dabrafenib Clinical Pharmacology and Biopharmaceutics Review. https://www.accessdata.fda.gov/ drugsatfda_docs/nda/2013/202806Orig1s000ClinPharmR.pdf. Accessed 13 February 2020

174. Rousset M, Dutriaux C, Bosco-Lévy P, Prey S, Pham-Ledard A, Dousset L, Gérard E, Bouchet S, Canal-Raffin M, Titier K, Molimard M (2017) Trough dabrafenib plasma concentrations can predict occurrence of adverse events requiring dose reduction in metastatic melanoma. Clin Chim Acta 472:26-29. https://doi. org/10.1016/j.cca.2017.07.012

175. Food and Drug Administration. Center for Drug Evaluation and Research (2018) Dacomitinib Clinical Pharmacology and Biopharmaceutics Review. https://www.accessdata.fda.gov/ drugsatfda_docs/nda/2018/211288Orig1s000MultidisciplineR. pdf. Accessed 13 February 2020

176. Wang X, Roy A, Hochhaus A, Kantarjian HM, Chen TT, Shah NP (2013) Differential effects of dosing regimen on the safety and efficacy of dasatinib:Retrospective exposure-response analysis of a phase III study. Clin Pharmacol Adv Appl 5:85-97. https://doi. org/10.2147/cpaa.s42796

177. Ishida Y, Murai K, Yamaguchi K, Miyagishima T, Shindo M, Ogawa K, Nagashima T, Sato S, Watanabe R, Yamamoto S, Hirose T, Saitou S, Yonezumi M, Kondo T, Kato Y, Mochizuki N, Ohno K, Kishino S, Kubo K, Oyake T, Ito S (2016) Pharmacokinetics and pharmacodynamics of dasatinib in the chronic phase of newly diagnosed chronic myeloid leukemia. Eur J Clin Pharmacol 72:185-193. https://doi.org/10.1007/ s00228-015-1968-y

178. Mizuta S, Sawa M, Tsurumi H, Matsumoto K, Miyao K, Hara T, Takahashi T, Sakemura R, Kojima H, Kohno A, Oba MS, Morita S, Sakamoto J, Emi N (2018) Plasma concentrations of dasatinib have a clinical impact on the frequency of dasatinib dose reduction and interruption in chronic myeloid leukemia: an analysis of the DARIA 01 study. Int J Clin Oncol 23:980-988. https://doi.org/10. 1007/s10147-018-1300-9

179. Yoshitsugu H, Imai Y, Seriu T, Hiraoka M (2012) Markov Chain Monte Carlo Bayesian analysis for population pharmacokinetics of dasatinib in Japanese adult subjects with chronic myeloid leukemia and Philadelphia chromosome positive acute lymphoblastic leukemia. Japanese J Clin Pharmacol Ther 43:29-41

180. Food and Drug Administration. Center for Drug Evaluation and Research (2017) NDA Multi-Disciplinary Review and Evaluation NDA 209606 IDHIFA (enasidenib). https:/www.accessdata.fda. gov/drugsatfda_docs/nda/2017/209606Orig1s000MultidisciplineR. pdf. Accessed 06 April 2020

181. Food and Drug Administration. Center for Drug Evaluation and Research (2018) NDA Multi-Disciplinary Review and Evaluation NDA 210496 BRAFTOVI $^{\mathrm{TM}}$ (encorafenib). https://www. accessdata.fda.gov/drugsatfda_docs/nda/2018/ 210496Orig1s000MultidisciplineR.pdf. Accessed 12 February 2020

182. Food and Drug Administration. Center for Drug Evaluation and Research (2019) NDA/BLA Multi-Disciplinary Review and Evaluation NDA 212018 BALVERSA (erdafitinib). https:// www.accessdata.fda.gov/drugsatfda_docs/nda/2019/ 212018Orig1s000MultidisciplineR.pdf. Accessed 06 April 2020

183. Dosne AG, Valade E, Stuyckens K, Li LY, Ouellet D, PerezRuixo JJ (2020) Population Pharmacokinetics of Total and Free Erdafitinib in Adult Healthy Volunteers and Cancer Patients: Analysis of Phase 1 and Phase 2 Studies. J Clin Pharmacol 60: 515-527. https://doi.org/10.1002/jcph.1547

184. Food and Drug Administration. Center for Drug Evaluation and Research (2019) NDA Multi-Disciplinary Review and Evaluation. NDA 212327 Inrebic (fedratinib). https://www.accessdata.fda.gov/ drugsatfda_docs/nda/2019/212327Orig1s000MultidisciplineR.pdf. Accessed $\overline{13}$ February 2020

185. Zhang M, Xu CR, Shamiyeh E, Liu F, Yin JY, Von Moltke LL, Smith WB (2014) A randomized, placebo-controlled study of the pharmacokinetics, pharmacodynamics, and tolerability of the oral JAK2 inhibitor fedratinib (SAR302503) in healthy volunteers. J Clin Pharmacol 54:415-421. https://doi.org/10.1002/jcph.218

186. Ogasawara K, Zhou S, Krishna G, Palmisano M, Li Y (2019) Population pharmacokinetics of fedratinib in patients with myelofibrosis, polycythemia vera, and essential thrombocythemia. 
Cancer Chemother Pharmacol 84:891-898. https://doi.org/10. 1007/s00280-019-03929-9

187. Food and Drug Administration. Center for Drug Evaluation and Research (2018) Glasdegib Clinical Pharmacology and Biopharmaceutics Review. https://www.accessdata.fda.gov/ drugsatfda_docs/nda/2018/210656Orig1s000ClinPharmR.pdf. Accessed 06 April 2020

188. Lin S, Shaik N, Martinelli G, Wagner AJ, Cortes J, Ruiz-Garcia A (2019) Population Pharmacokinetics of Glasdegib in Patients With Advanced Hematologic Malignancies and Solid Tumors. J Clin Pharmacol. https://doi.org/10.1002/jcph.1556

189. Food and Drug Administration. Center for Drug Evaluation and Research (2013) Ibrutinib Clinical Pharmacology and Biopharmaceutics Review. https://www.accessdata.fda.gov/ drugsatfda_docs/nda/2013/205552Orig1s000ClinPharmR.pdf. Accessed $\overline{13}$ February 2020

190. Marostica E, Sukbuntherng J, Loury D, De Jong J, De Trixhe XW, Vermeulen A, De Nicolao G, O’Brien S, Byrd JC, Advani R, McGreivy J, Poggesi I (2015) Population pharmacokinetic model of ibrutinib, a Bruton tyrosine kinase inhibitor, in patients with B cell malignancies. Cancer Chemother Pharmacol 75:111-121. https://doi.org/10.1007/s00280-014-2617-3

191. Food and Drug Administration. Center for Drug Evaluation and Research (2018) NDA Multi-Disciplinary Review and Evaluation NDA 211192 Tibsovo (ivosidenib). https:/www.accessdata.fda. gov/drugsatfda_docs/nda/2018/211192Orig1s000MultidisciplineR. pdf. Accessed 06 April 2020

192. Gupta N, Labotka R, Liu G, Hui AM, Venkatakrishnan K (2016) Exposure-safety-efficacy analysis of single-agent ixazomib, an oral proteasome inhibitor, in relapsed/refractory multiple myeloma: dose selection for a phase 3 maintenance study. Investig New Drugs 34:338-346. https://doi.org/10.1007/s10637-016-0346-7

193. Gupta N, Yang H, Hanley MJ, Zhang S, Liu R, Kumar S, Richardson PG, Skacel T, Venkatakrishnan K (2017) Dose and Schedule Selection of the Oral Proteasome Inhibitor Ixazomib in Relapsed/Refractory Multiple Myeloma: Clinical and ModelBased Analyses. Target Oncol 12:643-654. https://doi.org/10. 1007/s11523-017-0524-3

194. Gupta N, Diderichsen PM, Hanley MJ, Berg D, van de Velde H, Harvey RD, Venkatakrishnan K (2017) Population Pharmacokinetic Analysis of Ixazomib, an Oral Proteasome Inhibitor, Including Data from the Phase III TOURMALINEMM1 Study to Inform Labelling. Clin Pharmacokinet 56:13551368. https://doi.org/10.1007/s40262-017-0526-4

195. Committee for Medicinal Products for Human Use (CHMP) European Medicines Agency (2015) Lenvima European public assessment report. https://www.ema.europa.eu/en/documents/ assessment-report/lenvima-epar-public-assessment-report_en.pdf. Accessed 13 February 2020

196. Hayato S, Schumaker R, Ferry J, Binder T, Ductus CE, Hussein Z (2018) Exposure-response analysis and simulation of lenvatinib safety and efficacy in patients with radioiodine-refractory differentiated thyroid cancer. Cancer Chemother Pharmacol 82:971978. https://doi.org/10.1007/s00280-018-3687-4

197. Tamai T, Hayato S, Hojo S, Suzuki T, Okusaka T, Ikeda K, Kumada H (2017) Dose Finding of Lenvatinib in Subjects With Advanced Hepatocellular Carcinoma Based on Population Pharmacokinetic and Exposure-Response Analyses. J Clin Pharmacol 57:1138-1147. https://doi.org/10.1002/jcph.917

198. Nagahama M, Ozeki T, Suzuki A, Sugino K, Niioka T, Ito K, Miura M (2019) Association of lenvatinib trough plasma concentrations with lenvatinib-induced toxicities in Japanese patients with thyroid cancer. Med Oncol 36:39. https://doi.org/10.1007/ s12032-019-1263-3

199. Gupta A, Jarzab B, Capdevila J, Shumaker R, Hussein Z (2016) Population pharmacokinetic analysis of lenvatinib in healthy subjects and patients with cancer. Br J Clin Pharmacol 81:1124 1133. https://doi.org/10.1111/bcp.12907

200. Committee for Medicinal Products for Human Use (CHMP) European Medicines Agency (2019) Lorviqua European public assessment report. https://www.ema.europa.eu/en/documents/ assessment-report/lorviqua-epar-public-assessment-report_en. pdf. Accessed 14 February 2020

201. Food and Drug Administration. Center for Drug Evaluation and Research (2018) NDA Multi-disciplinary Review and Evaluation NDA 210868 Lorlatinib. https://www.accessdata.fda.gov/ drugsatfda_docs/nda/2018/210868Orig1s000MultidisciplineR. pdf. Accessed 14 February 2020

202. Shaw AT, Felip E, Bauer TM, Besse B, Navarro A, Postel-Vinay S, Gainor JF, Johnson M, Dietrich J, James LP, Clancy JS, Chen J, Martini JF, Abbattista A, Solomon BJ (2017) Lorlatinib in nonsmall-cell lung cancer with ALK or ROS1 rearrangement: an international, multicentre, open-label, single-arm first-in-man phase 1 trial. Lancet Oncol 18:1590-1599. https://doi.org/10.1016/ s1470-2045(17)30680-0

203. Wang Y, Yin OQP, Graf P, Kisicki JC, Schran H, Green K (2008) Dose- and time-dependent pharmacokinetics of midostaurin in patients with diabetes mellitus. J Clin Pharmacol 48:763-775. https://doi.org/10.1177/0091270008318006

204. Committee for Medicinal Products for Human Use (CHMP) European Medicines Agency (2017) Rydapt European public assessment report. https://www.ema.europa.eu/en/documents/ assessment-report/rydapt-epar-public-assessment-report_en.pdf. Accessed 14 February 2020

205. Food and Drug Administration. Center for Drug Evaluation and Research Midostaurin Clinical Pharmacology and Biopharmaceutics Review. Accessed 2020-04-01

206. Yin O, Wang Y, Lanza C, Schimansky T, Balez S, Schran HF, Stone RM (2008) Pharmacokinetics (PK) and pharmacodynamics (PD) of midostaurin (PKC412) in patients with acute myeloid leukemia (AML). J Clin Oncol 26:7064. https://doi.org/10.1200/ jco.2008.26.15_suppl.7064

207. Yin OQP, Wang Y, Schran H (2008) A mechanism-based population pharmacokinetic model for characterizing time-dependent pharmacokinetics of midostaurin and its metabolites in human subjects. Clin Pharmacokinet 47:807-816. https://doi.org/10. 2165/0003088-200847120-00005

208. Gandhi L, Bahleda R, Tolaney SM, Kwak EL, Cleary JM, Pandya SS, Hollebecque A, Abbas R, Ananthakrishnan R, Berkenblit A, Krygowski M, Liang Y, Turnbull KW, Shapiro GI, Soria JC (2014) Phase I study of neratinib in combination with temsirolimus in patients with human epidermal growth factor receptor 2-dependent and other solid tumors. J Clin Oncol 32:6875. https://doi.org/10.1200/jco.2012.47.2787

209. Committee for Medicinal Products for Human Use (CHMP) European Medicines Agency (2018) Nerlynx European public assessment report. https://www.ema.europa.eu/en/documents/ assessment-report/nerlynx-epar-public-assessment-report_en.pdf. Accessed 14 February 2020

210. Food and Drug Administration. Center for Drug Evaluation and Research (2014) Nintedanib Clinical Pharmacology and Biopharmaceutics Review. https://www.accessdata.fda.gov/ drugsatfda_docs/nda/2014/205832Orig1s000ClinPharmR.pdf. Accessed $1 \overline{3}$ February 2020

211. Okamoto I, Kaneda H, Satoh T, Okamoto W, Miyazaki M, Morinaga R, Ueda S, Terashima M, Tsuya A, Sarashina A, Konishi K, Arao T, Nishio K, Kaiser R, Nakagawa K (2010) Phase I safety, pharmacokinetic, and biomarker study of BIBF 1120 , an oral triple tyrosine kinase inhibitor in patients with advanced solid tumors. Mol Cancer Ther 9:2825-2833. https://doi. org/10.1158/1535-7163.mct-10-0379 
212. Schmid U, Heinz K, Angele L, Claudia F, Freiwald M (2018) Population pharmacokinetics of nintedanib, an inhibitor of tyrosine kinases, in patients with non-small cell lung cancer or idiopathic pulmonary fibrosis. Cancer Chemother Pharmacol 81:89101. https://doi.org/10.1007/s00280-017-3452-0

213. Committee for Medicinal Products for Human Use (CHMP) European Medicines Agency (2014) Nintedanib European Public Assessment Report. https://www.ema.europa.eu/en/ documents/assessment-report/vargatef-epar-public-assessmentreport_en.pdf. Accessed 13 February 2020

214. Sandhu SK, Schelman WR, Wilding G, Moreno V, Baird RD, Miranda S, Hylands L, Riisnaes R, Forster M, Omlin A, Kreischer N, Thway K, Gevensleben H, Sun L, Loughney J, Chatterjee M, Toniatti C, Carpenter CL, Iannone R, Kaye SB, de Bono JS, Wenham RM (2013) The poly(ADP-ribose) polymerase inhibitor niraparib (MK4827) in BRCA mutation carriers and patients with sporadic cancer: A phase 1 dose-escalation trial. Lancet Oncol 14:882-892. https://doi.org/10.1016/s1470-2045(13) 70240-7

215. Wang J, Zhang Z-Y, Mirza MR, Gilbert L, Fabbro M, Tinker AV, Wang X, Redondo A, Berek JS, Woelber L, Pentikis HS, Moore KN, Lorusso D, Benigno B, Hazard SJ, Follana P, Rimel BJ, Matulonis UA, Agarwal S, Kansra V (2017) 933PDThe exposure-response relationship of niraparib in patients with gBRCAmut and non-gBRCAmut: Results from the ENGOTOV16/NOVA Trial. Ann Oncol 28. https://doi.org/10.1093/ annonc/mdx372.003

216. Food and Drug Administration. Center for Drug Evaluation and Research (2017) Niraparib Clinical Pharmacology and Biopharmaceutics Review. https://www.accessdata.fda.gov/ drugsatfda_docs/nda/2017/208447Orig1s000MultidisciplineR. pdf. Accessed 31 March 2020

217. Zhou D, Li J, Bui K, Learoyd M, Berges A, Milenkova T, AlHuniti N, Tomkinson H, Xu H (2019) Bridging Olaparib Capsule and Tablet Formulations Using Population Pharmacokinetic Meta-analysis in Oncology Patients. Clin Pharmacokinet 58: 615-625. https://doi.org/10.1007/s40262-018-0714-x

218. Zhou D, Li J, Learoyd M, Bui K, Berges A, Milenkova T, AlHuniti N, Tomkinson H, Xu H (2019) Efficacy and Safety Exposure-Response Analyses of Olaparib Capsule and Tablet Formulations in Oncology Patients. Clin Pharmacol Ther 105: 1492-1500. https://doi.org/10.1002/cpt.1338

219. Food and Drug Administration. Center for Drug Evaluation and Research (2014) Olaparib Clinical Pharmacology and Biopharmaceutics Review. https://www.accessdata.fda.gov/ drugsatfda_docs/nda/2014/206162Orig1s000ClinPharmR.pdf. Accessed 14 February 2020

220. Committee for Medicinal Products for Human Use (CHMP) European Medicines Agency (2015) Osimertinib European Public Assessment Report. https://www.ema.europa.eu/en/ documents/assessment-report/tagrisso-epar-public-assessmentreport_en.pdf. Accessed 13 February 2020

221. Food and Drug Administration. Center for Drug Evaluation and Research (2015) Osimertinib Clinical Pharmacology and Biopharmaceutics Review. https://www.accessdata.fda.gov/ drugsatfda_docs/nda/2015/208065Orig1s000ClinPharmR.pdf. Accessed $1 \overline{3}$ February 2020

222. Brown K, Comisar C, Witjes H, Maringwa J, de Greef R, Vishwanathan K, Cantarini M, Cox E (2017) Population pharmacokinetics and exposure-response of osimertinib in patients with non-small cell lung cancer. Br J Clin Pharmacol 83:1216-1226. https://doi.org/10.1111/bcp.13223

223. Food and Drug Administration. Center for Drug Evaluation and Research (2014) Palbociclib Clinical Pharmacology and Biopharmaceutics Review. https://www.accessdata.fda.gov/
drugsatfda_docs/nda/2015/207103Orig1s000ClinPharmR.pdf. Accessed $1 \overline{2}$ February 2020

224. Flaherty KT, LoRusso PM, DeMichele A, Abramson VG, Courtney R, Randolph SS, Shaik MN, Wilner KD, O'Dwyer PJ, Schwartz GK (2012) Phase I, dose-escalation trial of the oral cyclin-dependent kinase 4/6 inhibitor PD 0332991, administered using a 21-day schedule in patients with advanced cancer. Clin Cancer Res 18:568-576. https://doi.org/10.1158/1078-0432.ccr11-0509

225. Sun W, O’Dwyer PJ, Finn RS, Ruiz-Garcia A, Shapiro GI, Schwartz GK, DeMichele A, Wang D (2017) Characterization of Neutropenia in Advanced Cancer Patients Following Palbociclib Treatment Using a Population PharmacokineticPharmacodynamic Modeling and Simulation Approach. J Clin Pharmacol 57:1159-1173. https://doi.org/10.1002/jcph.902

226. Van Veggel M, Westerman E, Hamberg P (2018) Clinical Pharmacokinetics and Pharmacodynamics of Panobinostat. Clin Pharmacokinet 57:21-29. https://doi.org/10.1007/s40262-0170565-X

227. Mu S, Kuroda Y, Shibayama H, Hino M, Tajima T, Corrado C, Lin R, Waldron E, Binlich F, Suzuki K (2016) Panobinostat PK/ $\mathrm{PD}$ profile in combination with bortezomib and dexamethasone in patients with relapsed and relapsed/refractory multiple myeloma. Eur J Clin Pharmacol 72:153-161. https://doi.org/10.1007/ s00228-015-1967-z

228. Savelieva M, Woo MM, Schran H, Mu S, Nedelman J, Capdeville R (2015) Population pharmacokinetics of intravenous and oral panobinostat in patients with hematologic and solid tumors. Eur J Clin Pharmacol 71:663-672. https://doi.org/10.1007/s00228015-1846-7

229. Food and Drug Administration. Center for Drug Evaluation and Research (2019) NDA/BLA Multi-disciplinary Review and Evaluation NDA 211810 TURALIO (pexidartinib). https:/www. accessdata.fda.gov/drugsatfda_docs/nda/2019/ 211810Orig1s000MultidisciplineR.pdf. Accessed 06 April 2020

230. Cortes JE, Kantarjian H, Shah NP, Bixby D, Mauro MJ, Flinn I, O'Hare T, Hu S, Narasimhan NI, Rivera VM, Clackson T, Turner CD, Haluska FG, Druker BJ, Deininger MWN, Talpaz M (2012) Ponatinib in refractory Philadelphia chromosome-positive leukemias. N Engl J Med 367:2075-2088. https://doi.org/10.1056/ nejmoa1205127

231. Food and Drug Administration. Center for Drug Evaluation and Research (2012) Ponatinib Clinical Pharmacology and Biopharmaceutics Review. https://www.accessdata.fda.gov/ drugsatfda_docs/nda/2012/203469Orig1s000ClinPharmR.pdf. Accessed 13 February 2020

232. Food and Drug Administration. Center for Drug Evaluation and Research (2012) Regorafenib Clinical Pharmacology and Biopharmaceutics Review. https://www.accessdata.fda.gov/ drugsatfda_docs/nda/2012/203085Orig1s000ClinPharmR.pdf. Accessed $1 \overline{2}$ February 2020

233. Committee for Medicinal Products for Human Use (CHMP) European Medicines Agency (2014) CHMP Regorafenib Extension of Indication Variation Assessment Report. https:// www.ema.europa.eu/en/documents/variation-report/stivarga-h-c2573-ii-0001-epar-assessment-report-variation_en.pdf. Accessed 12 February 2020

234. Suzuki T, Sukawa Y, Imamura CK, Masuishi T, Satake H, Kumekawa Y, Funakoshi S, Kotaka M, Horie Y, Kawai S, Okuda H, Terazawa T, Kondoh C, Kato K, Yoshimura K, Ishikawa H, Hamamoto Y, Boku N, Takaishi H, Kanai T (2020) A Phase II Study of Regorafenib With a Lower Starting Dose in Patients With Metastatic Colorectal Cancer: Exposure-Toxicity Analysis of Unbound Regorafenib and Its Active Metabolites (RESET Trial). Clin Colorectal Cancer 19:13-21.e3. https://doi. org/10.1016/j.clcc.2019.10.004 
235. Committee for Medicinal Products for Human Use (CHMP) European Medicines Agency (2013) Regorafenib European Public Assessment Report. https://www.ema.europa.eu/en/ documents/assessment-report/stivarga-epar-public-assessmentreport en.pdf.

236. Food and Drug Administration. Center for Drug Evaluation and Research (2017) NDA/BLA multi-disciplinary review and evaluation NDA 209092 KISQALI (ribociclib). https://www. accessdata.fda.gov/drugsatfda_docs/nda/2017/ 209092Orig1s000MultidisciplineR.pdf. Accessed 13 February 2020

237. Food and Drug Administration. Center for Drug Evaluation and Research (2016) NDA/BLA multi-disciplinary review and evaluation NDA 209115 Rubraca (rucaparib). https://www.accessdata.fda.gov/ drugsatfda_docs/nda/2016/209115Orig1s000MultiDisciplineR.pdf. Accessed 13 February 2020

238. Committee for Medicinal Products for Human Use (CHMP) European Medicines Agency (2018) Rubraca European public assessment report. https://www.ema.europa.eu/en/documents/ assessment-report/rubraca-epar-public-assessment-report_en.pdf.

239. Food and Drug Administration. Center for Drug Evaluation and Research. (2011) Ruxolitinib clinical pharmacology and biopharmaceutics review. https://www.accessdata.fda.gov/ drugsatfda_docs/nda/2011/202192Orig1s000ClinPharmR.pdf. Accessed 13 February 2020

240. Chen X, Williams WV, Sandor V, Yeleswaram S (2013) Population pharmacokinetic analysis of orally-administered ruxolitinib (incb018424 phosphate) in patients with primary myelofibrosis (pmf), post-polycythemia vera myelofibrosis (PPVMF) or post-essential thrombocythemia myelofibrosis (PET MF). J Clin Pharmacol 53:721-730. https://doi.org/10.1002/ jcph.102

241. Minami H, Kawada K, Ebi H, Kitagawa K, Kim YI1, Araki K, Mukai H, Tahara M, Nakajima H, Nakajima K (2008) Phase I and pharmacokinetic study of sorafenib, an oral multikinase inhibitor, in Japanese patients with advanced refractory solid tumors. Cancer Sci 99:1492-1498. https://doi.org/10.1111/j.1349-7006.2008. 00837.x

242. Shimada M, Okawa H, Kondo Y, Maejima T, Kataoka Y, Hisamichi K, Maekawa M, Matsuura M, Jin Y, Mori M, Suzuki H, Shimosegawa T, Mano N (2015) Monitoring serum levels of sorafenib and its $\mathrm{N}$-oxide is essential for long-term sorafenib treatment of patients with hepatocellular carcinoma. Tohoku J Exp Med 237:173-182. https://doi.org/10.1620/tjem.237.173

243. Fukudo M, Ito T, Mizuno T, Shinsako K, Hatano E, Uemoto S, Kamba T, Yamasaki T, Ogawa O, Seno H, Chiba T, Matsubara K (2014) Exposure-toxicity relationship of sorafenib in Japanese patients with renal cell carcinoma and hepatocellular carcinoma. Clin Pharmacokinet 53:185-196. https://doi.org/10.1007/s40262-0130108-Z

244. Boudou-Rouquette P, Ropert S, Mir O, Coriat R, Billemont B, Tod M, Cabanes L, Franck N, Blanchet B, Goldwasser F (2012) Variability of sorafenib toxicity and exposure over time: a pharmacokinetic/pharmacodynamic analysis. Oncologist 17: 1204-1212. https://doi.org/10.1634/theoncologist.2011-0439

245. Boudou-Rouquette P, Narjoz C, Golmard JL, ThomasSchoemann A, Mir O, Taieb F, Durand JP, Coriat R, Dauphin A, Vidal M, Tod M, Loriot MA, Goldwasser F, Blanchet B (2012) Early sorafenib-induced toxicity is associated with drug exposure and UGTIA9 genetic polymorphism in patients with solid tumors: a preliminary study. PLoS One 7:1-9. https://doi. org/10.1371/journal.pone.0042875

246. Blanchet B, Billemont B, Cramard J, Benichou AS, Chhun S, Harcouet L, Ropert S, Dauphin A, Goldwasser F, Tod M (2009) Validation of an HPLC-UV method for sorafenib determination in human plasma and application to cancer patients in routine clinical practice. J Pharm Biomed Anal 49:1109-1114. https://oi.org/10. 1016/j.jpba.2009.02.008

247. Jain L, Woo S, Gardner ER, Dahut WL, Kohn EC, Kummar S, Mould DR, Giaccone G, Yarchoan R, Venitz J, Figg WD (2011) Population pharmacokinetic analysis of sorafenib in patients with solid tumours. Br J Clin Pharmacol 72: 294-305. https://doi.org/ 10.1111/j.1365-2125.2011.03963.x

248. Committee for Medicinal Products for Human Use (CHMP) European Medicines Agency (2019) Talzenna European public assessment report. https://www.ema.europa.eu/en/documents/ assessment-report/talzenna-epar-public-assessment-report_en. pdf. Accessed 14 February 2020

249. Food and Drug Administration. Center for Drug Evaluation and Research (2018) NDA/BLA multi-disciplinary review and evaluation NDA 211651 TALZENNA (talazoparib). https://www. accessdata.fda.gov/drugsatfda_docs/nda/2018/ 211651Orig1s000MultidisciplineR.pdf. Accessed 14 February 2020

250. Yu Y, Durairaj C, Shi H, Wang DD (2020) Population pharmacokinetics of talazoparib in patients with advanced cancer. J Clin Pharmacol 60:218-228. https://doi.org/10.1002/jcph.1520

251. Committee for Medicinal Products for Human Use (CHMP) European Medicines Agency (2017) Tivozanib European public assessment report. https://www.ema.europa.eu/en/documents/ assessment-report/fotivda-epar-public-assessment-report_en.pdf.

252. Food and Drug Administration. Center for Drug Evaluation and Research. (2011) Vandetanib clinical pharmacology and biopharmaceutics review. https://www.accessdata.fda.gov/ drugsatfda_docs/nda/2011/022405Orig1s000ClinPharmR.pdf. Accessed $1 \overline{3}$ February 2020

253. Holden SN, Eckhardt SG, Basser R, de Boer R, Rischin D, Green M, Rosenthal MA, Wheeler C, Barge A, Hurwitz HI (2005) Clinical evaluation of ZD6474, an orally active inhibitor of VEGF and EGF receptor signaling, in patients with solid, malignant tumors. Ann Oncol 16:1391-1397. https://doi.org/10.1093/ annonc/mdi247

254. Tyrrell C, Denis L, Newling D, Soloway M, Channer K, Cockshott I (1998) Casodex TM 10-200 mg daily, used as monotherapy for the treatment of patients with advanced prostate cancer. Eur Urol 33:39-53

255. Saltzstein D, Sieber P, Morris T, Gallo J (2005) Prevention and management of bicalutamide-induced gynecomastia and breast pain: randomized endocrinologic and clinical studies with tamoxifen and anastrozole. Prostate Cancer Prostatic Dis 8:75-83. https://doi.org/10.1038/sj.pcan.4500782

256. Food and Drug Administration. Center for Drug Evaluation and Research. (2008) Bicalutamide clinical pharmacology and biopharmaceutics review. https://www.accessdata.fda.gov/ drugsatfda_docs/nda/2008/022310s000_ClinPharmR.pdf. Accessed 14 February 2020

257. Food and Drug Administration. Center for Drug Evaluation and Research. (2015) Cobimetinib clinical pharmacology and biopharmaceutics review. https://www.accessdata.fda.gov/ drugsatfda_docs/nda/2015/206192Orig1s000ClinPharmR.pdf. Accessed $1 \overline{2}$ February 2020

258. Han K, Jin JY, Marchand M, Eppler S, Choong N, Hack SP, Tikoo N, Bruno R, Dresser M, Musib L, Budha NR (2015) Population pharmacokinetics and dosing implications for cobimetinib in patients with solid tumors. Cancer Chemother Pharmacol 76:917-924. https://doi.org/10.1007/s00280-0152862-0

259. Food and Drug Administration. Center for Drug Evaluation and Research (2019) NDA multi-disciplinary review and evaluation NDA 212099 NUBEQA (darolutamide). https://www. accessdata.fda.gov/drugsatfda docs/nda/2019/ 212099Orig1s000MultidisciplineR.pdf. Accessed 06 April 2020 
260. Flinn IW, O’Brien S, Kahl B, Patel M, Oki Y, Foss FF, Porcu P, Jones J, Burger JA, Jain N, Kelly VM, Allen K, Douglas M, Sweeney J, Kelly P, Horwitz S (2018) Duvelisib, a novel oral dual inhibitor of PI3K-d,g, is clinically active in advanced hematologic malignancies. Blood 131:877-887. https://doi.org/10.1182/blood2017-05-786566

261. Food and Drug Administration. Center for Drug Evaluation and Research (2018) NDA multi-disciplinary review and evaluation NDA 211155 COPIKTRA (duvelisib). https://www.accessdata.fda. g o v/d r u g s a t f d a d o c s / n d a / $2018 /$ 211155Orig1Orig2s000MultidisciplineR.pdf. Accessed 13 February 2020

262. Food and Drug Administration. Center for Drug Evaluation and Research (2019) NDA/BLA multi-disciplinary review and evaluation NDA 212725 ROZLYTREK (entrectinib). https://www. accessdata.fda.gov/drugsatfda_docs/nda/2019/212725Orig1s000, 212726Orig1s000MultidisciplineR.pdf. Accessed 14 February 2020

263. Food and Drug Administration. Center for Drug Evaluation and Research. (2012) Enzalutamide clinical pharmacology and biopharmaceutics review. https://www.accessdata.fda.gov/ drugsatfda_docs/nda/2012/203415Orig1s000ClinPharmR.pdf. Accessed 12 February 2020

264. van Nuland M, Bergman AM, Rosing H, de Vries N, Huitema ADR, Beijnen JH (2019) Exposure-response assessment of enzalutamide and its major metabolites in a real-world cohort of patients with metastatic castration-resistant prostate cancer. Pharmacotherapy 39:1137-1145. https://doi.org/10.1002/phar. 2339

265. Valle M, Salle E Di, Jannuzzo MG, Poggesi I, Rocchetti M, Spinelli R, Verotta D (2005) A predictive model for exemestane pharmacokinetics / pharmacodynamics incorporating the effect of food and formulation. Br J Clin Pharmacol 59:3:355-364. https:// doi.org/10.1111/j.1365-2125.2005.02335.x

266. Food and Drug Administration. Center for Drug Evaluation and Research. (2014) Idelalisib clinical pharmacology and biopharmaceutics review. https://www.accessdata.fda.gov/ drugsatfda_docs/nda/2014/206545Orig1s000ClinPharmR.pdf. Accessed $1 \overline{4}$ February 2020

267. Sharma S, Guo Y, Jin F, Li X, Dubowy RL, Newcomb T, Ramanathan S (2015) Exposure-response of idelalisib administered in combination with ofatumumab for the treatment of relapsed chronic lymphocytic leukemia. Blood 126:4172 LP4174172

268. Daryani VM, Sharma S, Xing G, Silverman J, Adewoye AH, Mathias A (2017) Exposure-response of idelalisib administered in combination with bendamustine and rituximab for the treatment of relapsed chronic lymphocytic leukemi. Blood 130:5344 LP5345344

269. Jin F, Gao Y, Zhou H, Fang L, Li X (2016) Population pharmacokinetic modeling of idelalisib, a novel PI3K $\delta$ inhibitor, in healthy subjects and patients with hematologic malignancies. Cancer Chemother Pharmacol 77:89-98. https://doi.org/10.1007/ s00280-015-2891-8

270. Burris HA, Hurwitz HI, Dees EC, Dowlati A, Blackwell KL, O’Neil B, Marcom PK, Ellis MJ, Overmoyer B, Jones SF, Harris JL, Smith DA, Koch KM, Stead A, Mangum S, Spector NL (2005) Phase I safety, pharmacokinetics, and clinical activity study of lapatinib (GW572016), a reversible dual inhibitor of epidermal growth factor receptor tyrosine kinases, in heavily pretreated patients with metastatic carcinomas. J Clin Oncol 23: 5305-5313. https://doi.org/10.1200/jco.2005.16.584

271. Siegel-Lakhai WS, Beijnen JH, Vervenne WL, Boot H, Keessen M, Versola M, Koch KM, Smith DA, Pandite L, Richel DJ, Schellens JHM (2007) Phase I pharmacokinetic study of the safety and tolerability of lapatinib (GW572016) in combination with oxaliplatin/fluorouracil/leucovorin (FOLFOX4) in patients with solid tumors. Clin Cancer Res 13:4495-4502. https://doi.org/10. 1158/1078-0432.ccr-07-0004

272. Zhang J, Koch K (2012) Population pharmacokinetics of Lapatinib in cancer patients. PAGE Meet. https://doi.org/10. 1007/s40262-016-0452-x

273. Committee for Medicinal Products for Human Use (CHMP) European Medicines Agency (2019) Vitrakvi European public assessment report. https://www.ema.europa.eu/en/documents/ assessment-report/vitrakvi-epar-public-assessment-report_en.pdf.

274. Food and Drug Administration. Center for Drug Evaluation and Research (2018) NDA multi-disciplinary review and evaluation NDA 210861 and NDA 211710 VITRAKVI (larotrectinib). https://www.accessdata.fda.gov/drugsatfda_docs/nda/2018/ 210861Orig1s000 211710Orig1s000MultidisciplineR.pdf. Accessed 14 February 2020

275. Reda G, Cassin R, Dovrtelova G, Matteo C, Giannotta J, D'incalci M, Cortelezzi A, Zucchetti M (2019) Venetoclax penetrates in cerebrospinal fluid and may be effective in chronic lymphocytic leukemia with central nervous system involvement. Haematologica 104:e222-e223. https://doi.org/10.3324/ haematol.2018.213157

276. Food and Drug Administration. Center for Drug Evaluation and Research. (2016) Venetoclax clinical pharmacology and biopharmaceutics review. https://www.accessdata.fda.gov/ drugsatfda_docs/nda/2016/208573Orig1s000ClinPharmR.pdf. Accessed 13 February 2020

277. Jones AK, Freise KJ, Agarwal SK, Humerickhouse RA, Wong SL, Salem AH (2016) Clinical predictors of venetoclax pharmacokinetics in chronic lymphocytic leukemia and non-Hodgkin's lymphoma patients: a pooled population pharmacokinetic analysis. AAPS J 18:1192-1202. https://doi.org/10.1208/s12248-0169927-9

278. Food and Drug Administration. Center for Drug Evaluation and Research. (2012) Vismodegib clinical pharmacology and biopharmaceutics review. https:/www.accessdata.fda.gov/ drugsatfda docs/nda/2012/203388Orig1s000ClinPharmR.pdf. Accessed $1 \overline{2}$ Februrary 2020

279. Fujiwara Y, Yamamoto N, Yamada Y, Yamada K, Otsuki T, Kanazu S, Iwasa T, Hardwick JS, Tamura T (2009) Phase I and pharmacokinetic study of vorinostat (suberoylanilide hydroxamic acid) in Japanese patients with solid tumors. Cancer Sci 100: 1728-1734. https://doi.org/10.1111/j.1349-7006.2009.01237.x

280. Food and Drug Administration. Center for Drug Evaluation and Research (2006) Vorinostat clinical pharmacology and biopharmaceutics review. https://www.accessdata.fda.gov/ drugsatfda_docs/nda/2006/021991s000_Zolinza_ClinPharmR. pdf. Accessed 01 April 2020

281. Hertz DL, Kidwell KM, Seewald NJ, Gersch CL, Desta Z, Flockhart DA, Storniolo AM, Stearns V, Skaar TC, Hayes DF, Henry NL, Rae JM (2017) Polymorphisms in drug-metabolizing enzymes and steady-state exemestane concentration in postmenopausal patients with breast cancer. Pharmacogenomics J 17:521527. https://doi.org/10.1038/tpj.2016.60

282. Burris HA, Taylor CW, Jones SF, Koch KM, Versola MJ, Arya N, Fleming RA, Smith DA, Pandite L, Spector N, Wilding G (2009) A phase I and pharmacokinetic study of oral lapatinib administered once or twice daily in patients with solid malignancies. Clin Cancer Res 15:6702-6708. https://doi.org/10.1158/1078-0432. ccr-09-0369

Publisher's note Springer Nature remains neutral with regard to jurisdictional claims in published maps and institutional affiliations. 